\title{
A unified method for estimating pressure losses at vascular junctions
}

\author{
J. P. Mynard ${ }^{1,2,3 *}$, K. Valen-Sendstad ${ }^{1,4}$ \\ ${ }^{1}$ Biomedical Simulation Laboratory, Department of Mechanical and Industrial Engineering, University of Toronto, \\ Toronto ON, Canada \\ ${ }^{2}$ Heart Research, Clinical Sciences, Murdoch Childrens Research Institute, Parkville, VIC, Australia \\ ${ }^{3}$ Department of Paediatrics, University of Melbourne, Parkville, VIC, Australia \\ ${ }^{4}$ Center for Biomedical Computing, Simula Research Laboratory, Lysaker, Norway
}

\begin{abstract}
SUMMARY
In reduced order (0D/1D) blood or respiratory flow models, pressure losses at junctions are usually neglected. However, these may become important where velocities are high and significant flow redirection occurs. Current methods for estimating losses rely on relatively complex empirical equations that are only valid for specific junction geometries and flow regimes. In pulsatile multi-directional flows, switching between empirical equations upon reversing flow may introduce unrealistic discontinuities in simulated haemodynamic waveforms. Drawing from work by Bassett et al (SAE Trans 112:565-583, 2003), we therefore developed a unified method (Unified0D) for estimating loss coefficients that can be applied to any junction (i.e. any number of branches at any angle) and any flow regime. Discontinuities in simulated waveforms were avoided by extending Bassett et al's control volume-based method to incorporate a 'pseudodatum' supplier branch, an imaginary effective vessel containing all inflow to the junction. Energy exchange between diverging flow streams was also accounted for empirically. The formulation was validated using high resolution computational fluid dynamics in a wide range flow conditions and junction configurations. In a pulsatile 1D simulation exhibiting transitions between four different flow regimes, the new formulation produced smooth transitions in calculated pressure losses. Copyright (c) 2010 John Wiley \& Sons, Ltd.
\end{abstract}

Received . .

KEY WORDS: blood flow, respiratory flow, one-dimensional model, lumped parameter model, loss coefficient, bifurcation

\section{INTRODUCTION}

Reduced order models of flow in tube networks are widely used in physiological research. With one-dimensional (1D) blood flow modelling, the spatial evolution of pressure and flow waveforms can be predicted with minimal computational effort $(1 ; 2 ; 3 ; 4)$. Lumped parameter (0D) models can also be used to represent vascular networks, either explicitly at different levels of detail $(5 ; 6 ; 7)$, or implicitly by making use of (sub)network fractal properties $(8 ; 9 ; 10 ; 11)$. Similar models have been applied to study flow in airways $(12 ; 13 ; 14)$. In all such models, sectionally-averaged pressure and flow/velocity are calculated in individual segments via the $0 \mathrm{D}$ or 1D governing equations, while the communication between segments is handled at branch junctions with two interface conditions. The first condition enforces mass conservation, while the second governs conservation of total

\footnotetext{
${ }^{*}$ Correspondence to: J.P. Mynard, Heart Research, Clinical Sciences, Murdoch Childrens Research Institute, 50 Flemington Rd. Parkville, VIC 3052, Australia. Email: jonathan.mynard@mcri.edu.au
} 
mechanical energy. Formulation of this latter condition is not straightforward because pressure losses occur in the vicinity of junctions, arising from complex three-dimensional (3D) flow patterns that depend on factors such as flow distribution, branch area ratios, branch angles, and specific junction geometry.

In the context of $1 \mathrm{D}$ blood flow modelling, junction pressure losses have been treated in various ways. Many investigators $(15 ; 1 ; 16 ; 3 ; 17 ; 18)$ have assumed the losses to be negligible and have thus employed continuity of total pressure at all junctions $\left(\hat{p}=p+\frac{1}{2} \rho u^{2}\right.$, where $p$ is static pressure, $\hat{p}$ is dynamic pressure, $\rho$ is fluid density and $u$ is velocity). Others have chosen continuity of static pressure $(19 ; 9 ; 2)$ which assumes that any excess dynamic pressure in one branch compared to another is dissipated completely. In instances where non-negligible losses have been anticipated $(20 ; 21)$, loss coefficients have been estimated from empirical equations that are specific to particular junction geometries (e.g. T-junction) and flow regimes (22; 23).

Although pressure losses at vascular junctions are usually assumed to be small, even small losses may have an appreciable cumulative effect in large vascular trees containing numerous junctions. Indeed, the relevance of the branch formulation was highlighted by San et al (24), who found differences of up to $8 \mathrm{mmHg}$ and $20 \mathrm{~mL} / \mathrm{s}$ in simulated peak pressure and flow when assuming continuity of total versus static pressure at junctions in a model of the 55 largest systemic arteries. Moreover, in normal, pathological or surgically-induced situations where high velocity flows are encountered (e.g. exercise, the fetal ductus arteriosus, collaterals, stenoses, bypass grafts or arterio-venous anastomoses), losses at individual junctions may also be important (20;25). Various empirical equations exist for estimating junction loss coefficients in reduced order models, but these can only be applied to specific junction configurations and flow conditions. In addition, we have found that switching between such equations when flow conditions change dynamically can lead to non-physiological discontinuities in the simulated pressure/flow waveforms.

Drawing from the formulation described by Bassett et al (26), the aim of this study was to develop and validate a unified method for estimating pressure losses in any vascular junction and for any flow condition. The code for this 'Unified0D' method is being made publicly available and can therefore be easily incorporated into existing $1 \mathrm{D}$ codes. The method leads to smooth solutions in the presence of flow reversals. Calculated losses are validated against high resolution computational fluid dynamics (CFD) simulations over a wide range of junction geometries and flow conditions.

\section{METHODS}

\subsection{Junction flow classification}

Flow patterns and associated pressure losses in tube junctions are determined by geometry (tube shapes, tube sizes, junction angles, degree of filleting and number of branches) and flow characteristics (flow magnitude, flow ratios and inflow velocity profiles). Although there are clearly an infinite number of possible configurations, in this paper we test a wide (albeit not comprehensive) sampling of these configurations. Since our primary interest is physiological flow, we only consider circular tubes typical of blood vessels and airways. In addition, although the formulation for estimating loss coefficients can be robustly applied to junctions with any number of branches, we limit our validation tests to three-branch junctions.

To structure the validation tests, six junction/flow types are defined (Figure 1). Types 1 to 3 are diverging flows, while types 4 to 6 are converging flows. Types 1 and 4 are T-junctions in which only part of the flow enters or exits via the side branch, while in Types 2 and 5, all of the flow enters or exits via the side branch. Types 3 and 6 refer to diverging and converging flows in Y-junctions. Parameters sweeps were performed by holding all parameters constant except one. Unless otherwise stated, a flow fraction of 0.5 is used (i.e. flow divided equally between inlets for converging flow or between outlets for diverging flow), along with a branch angle of 90 degrees for T-junctions or 45 degrees for Y-junctions, and an area ratio of 1.0 (all tubes of equal diameter). Reynolds numbers $(R e)$ are specified in the figure captions. 

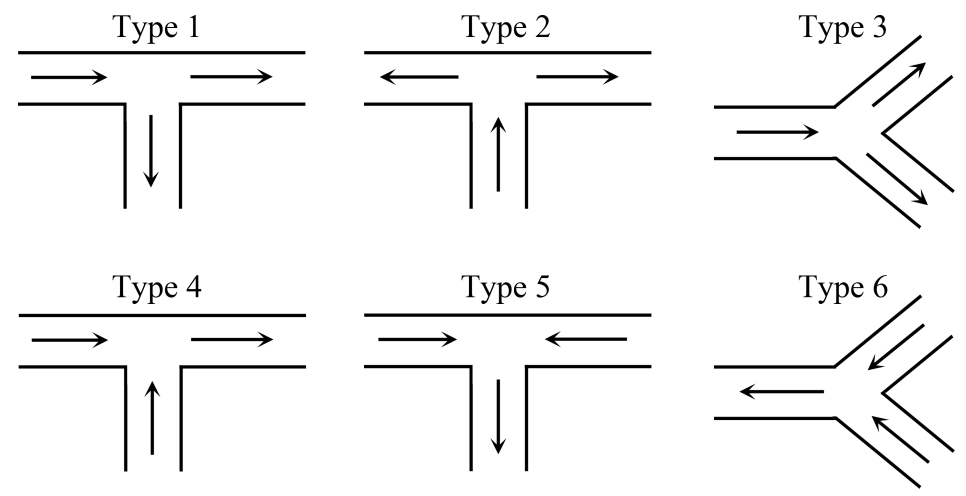

Figure 1. Definition of six classes of three-branch junction flows. Types 1 to 3 are diverging flows, while types 4 to 6 are converging flows.

\subsection{Computational fluid dynamics (CFD)}

Three-dimensional circular tube junctions were created in ANSYS $^{\circledR}{ }^{\circledR C E M ~ C F D ~}{ }^{\mathrm{TM}}$ (V1.21, Canonsburg, PA, USA) or Autodesk ${ }^{\circledR}$ Inventor ${ }^{\circledR}$ Professional 2014 (San Rafael, CA, USA). Inlet length was $20 \mathrm{~mm}$ and outlets were $>30$ diameters long. Sharp corners were smoothed slightly via the vmtksurfacesmoothing function in the Vascular Modelling Toolkit (VMTK, www.vmtk.org), with 10 iterations and a passband of 0.1; or, when testing the effect of filleting (Section 3.4), 6 $\mathrm{mm}$ fillets were added in Autodesk Inventor. Volumetric meshes generated in VMTK included two boundary layers and consisted of between 1.8 and 4.0 million linear tetrahedral elements, with a typical element edge length of $0.25 \mathrm{~mm}$ (near the junction) to $0.39 \mathrm{~mm}$ (far from the junction) for $6 \mathrm{~mm}$ diameter tubes. The no-slip condition was enforced on the rigid side walls and zero pressure was imposed on one outlet boundary. For the remaining inlets/outlets, a constant flow Dirichlet boundary condition was imposed with the following power-law velocity profile,

$$
U(r)=\left(\frac{\gamma+2}{\gamma}\right)\left[1-\left(\frac{r}{R}\right)^{\gamma}\right] U_{\text {mean }}
$$

where $R$ is the tube radius, $r$ is the radial coordinate and $U_{\text {mean }}$ is the cross-sectional mean velocity. Unless otherwise stated, $\gamma=9$ which corresponds to a profile commonly used in 1D blood flow models and is essentially flat for $r<0.6$, but falls to zero at the boundaries $(27 ; 28 ; 3 ; 29)$. Note that in Section 3.4 we test the sensitivity of the loss coefficients to this parameter (and hence to peak velocity independent of mean velocity) by also using $\gamma=2$ (i.e. a parabolic profile).

The transient Navier-Stokes equations were solved using Oasis, an open-source solver implemented in the finite element library FEniCS (30). The solver is a minimally dissipative 2nd order accurate (space and time) incremental pressure correction scheme shown to handle both laminar and turbulent flows. A detailed description of the solver is given in (31), but the key points are included here for completeness. In short, the Navier-Stokes equations are solved using an incremental pressure correction scheme (32). The governing equations are thus

$$
\begin{aligned}
\frac{\partial \boldsymbol{u}}{\partial t}+(\boldsymbol{u} \cdot \nabla) \boldsymbol{u} & =\nu \nabla^{2} \boldsymbol{u}-\nabla p+\boldsymbol{f}, \\
\nabla \cdot \boldsymbol{u} & =0
\end{aligned}
$$

where $\boldsymbol{u}(\boldsymbol{x}, t)$ is the velocity vector, $\nu$ the kinematic viscosity, $p(\boldsymbol{x}, t)$ the fluid pressure. Any volumetric forces are denoted by $\boldsymbol{f}(\boldsymbol{x}, t)$, and the constant fluid density is incorporated into the pressure.

Time is split up into uniform intervals using a constant time step $\triangle t=t^{n}-t^{n-1}$, where superscript $n$ is an integer and $t^{n} \in \mathcal{R}^{+}$. Discretization in space is performed using finite elements, whereas discretization in time is performed with finite differences. Following Simo and Armero 
(33) the fractional step algorithm can be written as

$$
\begin{gathered}
\frac{u_{k}^{I}-u_{k}^{n-1}}{\triangle \mathrm{t}}+B_{k}^{n-1 / 2}=\nu \nabla^{2} \tilde{u}_{k}-\nabla_{k} p^{*}+f_{k}^{n-1 / 2} \\
\text { for } k=1, \ldots, d \\
\nabla^{2} \varphi=-\frac{1}{\triangle \mathrm{t}} \nabla \cdot \boldsymbol{u}^{I} \\
\frac{u_{k}^{n}-u_{k}^{I}}{\triangle \mathrm{t}}=-\nabla_{k} \varphi \quad \text { for } k=1, \ldots, d
\end{gathered}
$$

where $u_{k}^{n}$ is component $k$ of the velocity vector at time $t^{n}, d$ is the dimension of the problem and $\varphi=p^{n-1 / 2}-p^{*}$ is a pressure correction. We are solving for the velocity and pressure on the next time step, i.e., $u_{k}^{n}$ for $k=1, \ldots, d$ and $p^{n-1 / 2}$. However, the tentative velocity equation (4) is solved with the tentative velocity component $u_{k}^{I}$ as unknown. To avoid strict time step restrictions and to maintain second order accuracy, the viscous term is discretized using a semi-implicit CrankNicolson interpolated velocity component $\tilde{u}_{k}=0.5\left(u_{k}^{I}+u_{k}^{n-1}\right)$. The nonlinear convection term is denoted by $B_{k}^{n-1 / 2}$, indicating that it should be evaluated at the midpoint between time steps $n$ and $n-1$. We have here used the semi-implicit

$$
B_{k}^{n-1 / 2}=\bar{u} \cdot \nabla \tilde{u}_{k},
$$

that is, an Adams-Bashforth projected convecting velocity vector $\overline{\boldsymbol{u}}=1.5 \boldsymbol{u}^{n-1}-0.5 \boldsymbol{u}^{n-2}$ and Crank-Nicolson for the convected velocity. The Adams-Bashforth discretization is second order accurate in time and, since the convecting velocity is known, there is no implicit coupling between the three velocity components solved for in the tentative velocity equations. This is a deliberate choice because it is important for the efficiency, and coupling is only effected through the pressure.

2.2.1. Calculation of reference loss coefficients To quantify pressure difference across a junction, cross-sectionally averaged pressures were first extracted from the 3D pressure field along the entire length of each tube in $1 \mathrm{~mm}$ steps, using a python script in Paraview (V4.1, Kitware, Inc.). The pressure drop in the vicinity of the junction was calculated using the method described by Gardel (22), as illustrated in Figure 2. Linear fits of the nearest $3 \mathrm{~cm}$ of stable declining pressure on either side of the junction were extrapolated to the junction centre point $(x=0)$ in a 1D space defined by the tube centrelines. The pressure difference $\Delta p_{i, j}$ between upstream branch $i$ and downstream branch $j$ was then calculated, along with the loss coefficient defined as

$$
K_{i, j}=\frac{\Delta p_{i, j}+\frac{1}{2} \rho\left(u_{i}-u_{j}\right)^{2}}{\frac{1}{2} \rho u_{\mathrm{com}}^{2}}
$$

where $u_{\text {com }}$ is velocity in the common branch (i.e. that contains all the inflow or outflow) and all velocities are cross-sectional mean values, calculated as volumetric flow divided by vessel cross-sectional area. $K_{i, j}$ is the reference loss coefficient ('Ref3D') against which reduced-order estimation methods are evaluated.

\subsection{One-dimensional model}

For a straight cylindrical vessel with deformable walls, the incompressible Navier-Stokes equations can be reduced to the following $1 \mathrm{D}$ equations (34),

$$
\begin{gathered}
\frac{\partial A}{\partial t}+\frac{\partial A u}{\partial x}=0 \\
\frac{\partial u}{\partial t}+u \frac{\partial u}{\partial x}+\frac{1}{\rho} \frac{\partial p}{\partial x}=f
\end{gathered}
$$




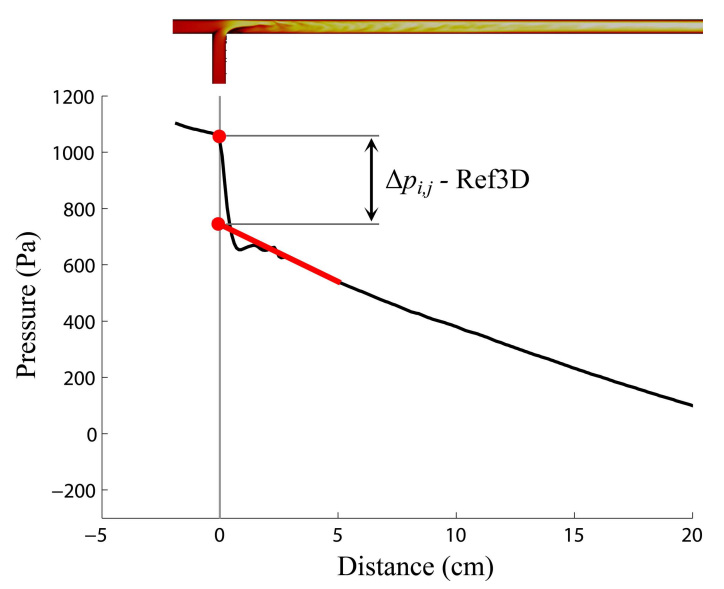

Figure 2. Method for calculating the junction pressure difference $\left(\Delta p_{i, j}\right)$ between branch $i$ and $j$ from CFD data. Inset at top shows the velocity magnitude on a centered axial slice.

where $u(x, t)$ and $p(x, t)$ are sectionally-averaged velocity and pressure, $A(x, t)$ is cross-sectional area, and $\rho$ is blood density. In the absence of body forces, $f$ represents viscous losses and is approximated by the expression $-2(\gamma+2) \pi \mu u / A$, where $\gamma$ determines the bluntness of the velocity profile via equation (1). Volumetric blood flow is calculated as $q=u A$. The system of equations is completed with a constitutive relation governing the fluid-structure interaction. In the context of blood flow modelling, this relation is non-linear and well approximated by a power law (35),

$$
p(A)-p_{\text {ext }}=\frac{2 \rho c_{0}^{2}}{b}\left[\left(\frac{A}{A_{0}}\right)^{b / 2}-1\right]+P_{0}
$$

where $p_{\text {ext }}$ is external pressure (assumed to be zero in this study) and $A_{0}$ and $c_{0}$ are reference crosssectional area and wave speed at reference pressure $P_{0}$. A physiological value of $b$ can be obtained by estimating a nominal vessel collapse pressure $P_{\text {collapse }}=\left.P\right|_{A=0}$ (35), giving

$$
b=\frac{2 \rho c_{0}^{2}}{\left(P_{0}-P_{\text {collapse }}\right)}
$$

\subsection{Formulation for estimating junction loss coefficients}

To represent the junction of two or more tube segments in a 1D (or 0D) model, mass and energy coupling conditions are required $(36 ; 37)$. Consider the junction of $n$ 1D branches, represented as a single point in 1D space. In this paper, branches containing flow entering the junction are called 'suppliers' and branches for which flow leaves the junction are called 'collectors'. Given a convention that supplier flow is positive and collector flow is negative, mass conservation is given by

$$
\sum_{n} q_{n}=0
$$

Continuity of total mechanical energy between any two branches $(i, j)$ is expressed as $(38 ; 26)$

$$
p_{i}+\frac{1}{2} \rho u_{i}^{2}=p_{j}+\frac{1}{2} \rho u_{j}^{2}+p_{\text {loss }}
$$

where $p$ and $\frac{1}{2} \rho u^{2}$ are the static and dynamic components of pressure respectively, and $\hat{p}=p+\frac{1}{2} \rho u^{2}$ is called total pressure (in this paper hydrostatic pressure is ignored). The pressure loss ( $\left.p_{\text {loss }}\right)$ 
represents energy dissipation ${ }^{\dagger}$ and for three-branch junctions is usually expressed in terms of kinetic energy in the 'common' branch that contains all of the flow $\left(\frac{1}{2} \rho u_{\mathrm{com}}^{2}\right)$ and a loss coefficient $\left(K_{i, j}\right)$ that depends on the junction geometry and flow conditions,

$$
p_{\text {loss }}=K_{i, j}\left(\frac{1}{2} \rho u_{\text {com }}^{2}\right)
$$

Given known upstream and downstream pressures, the loss coefficient can be calculated via equation (8). In Y or T junctions, the common branch is the single supplier for diverging flows and the single collector for converging flows. However, equation (15) cannot be applied to all junctions. For example, if a four-branch junction consists of two suppliers and two collectors, then a 'common' branch does not exist. An alternative coefficient $\left(C_{j}\right)$ that can be applied to any junction is defined according to the static pressure difference between upstream and downstream branches (26),

$$
p_{i}-p_{j}=C_{j}\left(\rho u_{j}^{2}\right)
$$

noting that the kinetic energy in collector $j$ is used, rather than that in a common branch. Substituting (16) into (14),

$$
p_{\text {loss }}=C_{j}\left(\rho u_{j}^{2}\right)+\frac{1}{2} \rho\left(u_{i}^{2}-u_{j}^{2}\right)
$$

For junctions in which a common branch exists, the two loss coefficients are related via

$$
C_{j}=\frac{1}{2}\left(K_{j} \frac{u_{\mathrm{com}}^{2}}{u_{j}^{2}}-\frac{u_{i}^{2}}{u_{j}^{2}}+1\right)
$$

Many existing 1D blood flow models have assumed continuity of total pressure at junctions, i.e. $K_{i, j}=0$ or $C_{j}\left(\rho u_{j}^{2}\right)=\frac{1}{2} \rho\left(u_{i}^{2}-u_{j}^{2}\right)(39 ; 16 ; 18)$. Others have assumed continuity of static pressure, which is equivalent to $K_{i, j}=\left(u_{i}^{2}-u_{j}^{2}\right) / u_{i}^{2}$ or $C_{j}=0$ (19; 38). Alternatively, loss coefficients in T-junctions may be estimated using the empirical equations described by Gardel $(40 ; 22)$ and Wood et al (23). However, these do not apply to Y-junctions or junctions with more than three branches. Bassett et al (26) described a formulation to estimate pressure losses in engine pipe networks, which in principle can be applied to any junction, i.e. any number of branches with arbitrary diameters joining at arbitrary angles and containing arbitrary flow ratios. However, as far as we are aware, no unified formulation has been described that is suitable for pulsatile flows in which reversing flow may cause the flow regime to change abruptly.

In following sections, we outline the original formulation described by Bassett et al (26) that is based on a control volume analysis, and then describe two refinements, namely 1) the introduction of a pseudodatum supplier branch to obtain smooth solutions for pulsatile multi-directional flow problems and 2) a modification to account for energy exchange between two or more collectors.

2.4.1. Control volume analysis The formulation described by Bassett et al (26) considers a junction of $n$ vessels in which one supplier containing all or most of the flow entering the junction is designated as the 'datum' branch (Figure 3). Following previous studies $(41 ; 42)$, continuity of static pressure is assumed between the datum branch and any other suppliers, while a loss coefficient is calculated between the datum branch and each collector.

To estimate the loss coefficient $\left(K_{\mathrm{dat}, j}\right)$ between the datum branch and collector $j$, Bassett et al (26) considered two control volumes, ABCD and CDEF indicated by dotted lines in Figure 3. Flow entering branch $j$ passes through $\mathrm{AB}$, separates from the wall $\mathrm{AE}$, contracts to $\mathrm{DC}$ and then expands to EF. Based on experimental data, Hager (43) estimated that the fluid crossing $\mathrm{AB}\left(u_{\mathrm{AB}}\right)$ deviates (on average along the line $\mathrm{AB}$ ) by approximately $\left(\pi-\phi_{j}\right) / 4$ from the datum branch axis $\left(\theta_{\text {dat }}\right)$. In regions of flow contraction there is little energy dissipation (43), and so the momentum equation for $\mathrm{ABCD}$ in the axial direction of branch $j$ is

\footnotetext{
$\dagger$ It should be noted, however, that $p_{\text {loss }}$ may also be affected by energy exchange between branches, as will be discussed in detail later.
} 


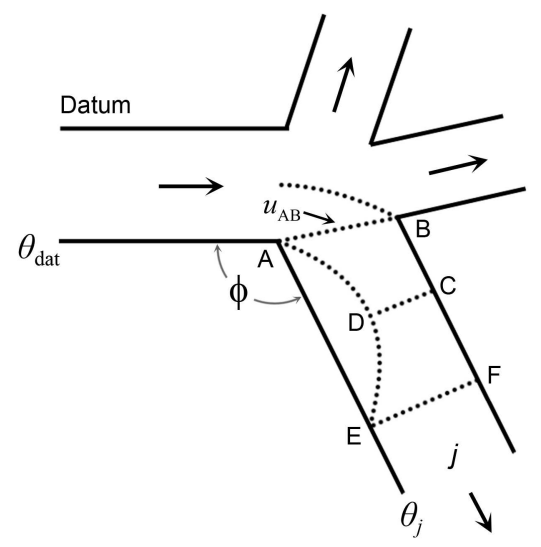

Figure 3. Schematic for the control volume analysis described by Bassett et al (26).

$$
A_{j}\left(p_{\mathrm{AB}}-p_{\mathrm{DC}}\right)=\rho q_{j}\left(u_{\mathrm{DC}}-u_{\mathrm{AB}} \cos \varphi_{j}\right)
$$

where $\varphi_{j}=\frac{3}{4}\left(\pi-\phi_{j}\right)$ is the angle between the $u_{\mathrm{AB}}$ vector and the axis of branch $j$, and

$$
\phi_{j}=\theta_{\mathrm{dat}}-\theta_{j} .
$$

The corresponding momentum equation for $\mathrm{CDEF}$ is

$$
A_{j}\left(p_{\mathrm{DC}}-p_{\mathrm{EF}}\right)=\rho q_{j}\left(u_{\mathrm{EF}}-u_{\mathrm{DC}}\right)
$$

Assuming that the overall pressure loss ( $\left.p_{\text {loss }}\right)$ occurs entirely as the flow diverges (43), Bernoulli's equation for $\mathrm{CDEF}$ is

$$
p_{\mathrm{DC}}+\frac{1}{2} \rho u_{\mathrm{DC}}^{2}=p_{\mathrm{EF}}+\frac{1}{2} \rho u_{\mathrm{EF}}^{2}+p_{\text {loss }}
$$

If flow ratio $\left(\lambda_{j}\right)$, area ratio $\left(\psi_{j}\right)$ and flow contraction ratio $(\xi)$ are defined as

$$
\lambda_{j}=\frac{q_{j}}{q_{\mathrm{dat}}} \quad, \quad \psi_{j}=\frac{A_{\mathrm{dat}}}{A_{j}} \quad, \quad \xi_{j}=\frac{A_{\mathrm{DC}}}{A_{j}}
$$

and if, following Hager (43) and Bassett et al (26), we assume that $u_{\mathrm{AB}} \approx u_{\mathrm{dat}}$, then

$$
u_{\mathrm{DC}}=\lambda_{j} \psi_{j} \frac{u_{\mathrm{dat}}}{\xi} \quad, \quad u_{\mathrm{EF}}=\lambda_{j} \psi_{j} u_{\mathrm{dat}}
$$

Substituting (21), (23) and (24) into (22) yields the following expression for $p_{\text {loss }}$,

$$
p_{\text {loss }}=\frac{1}{2} \rho \lambda_{j}^{2} \psi_{j}^{2} u_{\text {dat }}^{2}\left(1-\frac{1}{\xi}\right)^{2}
$$

We now seek to eliminate $\xi$ in (25). Assuming pressure at B is equal to stagnation pressure in the datum branch (i.e. $p_{\mathrm{B}}=\hat{p}_{\text {dat }}$ ), that $p_{\mathrm{A}}=p_{\text {dat }}$ and that the mean pressure along $\mathrm{AB}$ is the average of $p_{A}$ and $p_{B}$, then

$$
p_{\mathrm{AB}}=\frac{1}{2}\left(p_{\mathrm{A}}+p_{\mathrm{B}}\right)=p_{\mathrm{dat}}+\frac{1}{4} \rho u_{\mathrm{dat}}^{2}
$$

If no energy dissipation occurs in the region of flow contraction $(\mathrm{ABCD})$ and if the pressure and axial velocity are uniform over DC, then

$$
\hat{p}_{\mathrm{DC}}=\hat{p}_{\mathrm{AB}}=p_{\mathrm{dat}}+\frac{3}{4} \rho u_{\mathrm{dat}}^{2}=p_{\mathrm{DC}}+\frac{1}{2} \rho u_{\mathrm{DC}}^{2}
$$

Then by substituting (24), (26) and (27) into (19), it can be shown that

$$
\frac{1}{\xi}=1+\sqrt{1+\frac{1}{\lambda_{j}^{2} \psi_{j}^{2}}-\frac{2}{\lambda_{j} \psi_{j}} \cos \varphi_{j}}
$$


noting that $0<\xi \leq 1$. The pressure loss (25) can then be expressed purely in terms of vascular geometry $\left(\psi_{j}, \varphi_{j}\right)$, flow ratio $\left(\lambda_{j}\right)$ and velocity in the datum branch:

$$
p_{\text {loss }}=\frac{1}{2} \rho u_{d a t}^{2}\left(1+\lambda_{j}^{2} \psi_{j}^{2}-2 \lambda_{j} \psi_{j} \cos \varphi_{j}\right)
$$

The associated loss coefficient is

$$
C_{j}=1-\frac{1}{\lambda_{j} \psi_{j}} \cos \left[\frac{3}{4}\left(\pi-\varphi_{j}\right)\right]
$$

2.4.2. The pseudodatum branch Defining the datum branch as the single branch with the most flow entering the junction leads to two problems. First, if there are two or more suppliers with equal inflow, it is unclear which branch should be designated datum. Second, in pulsatile flow simulations in which the majority supplier changes from one instant to the next, we have found that abrupt switching of the assigned datum branch produces discontinuities in simulated pressure/flow waveforms.

To avoid these issues, we propose a refinement to the formulation described by Bassett et al (26), namely, replacing the datum branch with a 'pseudodatum branch', a virtual branch with an effective angle and diameter containing combined junction inflow. This idea was proposed by Bassett et al (26), but was not implemented since it was considered an unnecessary complication. However, while Bassett et al (26) considered applications involving unidirectional flows only, use of a pseudodatum branch is essential for obtaining smooth pressure/flow waveforms under pulsatile multi-directional flow conditions. Thus, hereafter the subscript 'dat' refers to the pseudodatum branch.

Flow in the pseudodatum branch is defined as

$$
q_{\mathrm{dat}}=\sum_{i \in S} q_{i}
$$

where $S$ is the set of suppliers. Velocity in the pseudodatum branch is the flow-weighted value from all suppliers, i.e.

$$
u_{\mathrm{dat}}=\frac{1}{q_{S}} \sum_{i \in S} q_{i} u_{i}
$$

where $q_{S}$ is total flow entering the junction. Pseudodatum area is then given by

$$
A_{\text {dat }}=\frac{q_{\text {dat }}}{u_{\text {dat }}}
$$

The pseudodatum branch angle is calculated as the flow-weighted average of supplier angles,

$$
\theta_{\mathrm{dat}}=\arctan \left(\frac{\sum_{i \in S} q_{i} \sin \theta_{i}}{\sum_{i \in S} q_{i} \cos \theta_{i}}\right)
$$

where the average is calculated via Cartesian transformation to avoid ambiguities arising from the circularity of angles (e.g. the average of 5 and 355 degrees is 0 rather than 180).

2.4.3. Accounting for energy exchange between collectors So far, it has been assumed that the loss coefficient for collector $j$ is solely dependent on the properties of that collector $\left(\varphi_{j}, \lambda_{j}, \psi_{j}\right)$, i.e. with no dependence on properties of other collectors. However, three findings from CFD studies suggested that such dependencies do exist, as will be discussed in more detail in later sections. In brief, the first was a finding of negative $K_{\text {dat }, j}$ values in some diverging flow cases. The second was that even when $\varphi_{j}$ was held constant, $K_{\mathrm{dat}, j}$ was altered by $\varphi_{k}$ (i.e. the angle of another collector). Third, in the case of diverging flow in a T-junction, changing the inlet velocity profile by decreasing $\gamma$ in equation (1) increased the loss coefficient in the side branch, but decreased it (i.e. made it more negative) in the straight branch. These observations suggest that a form of energy exchange 

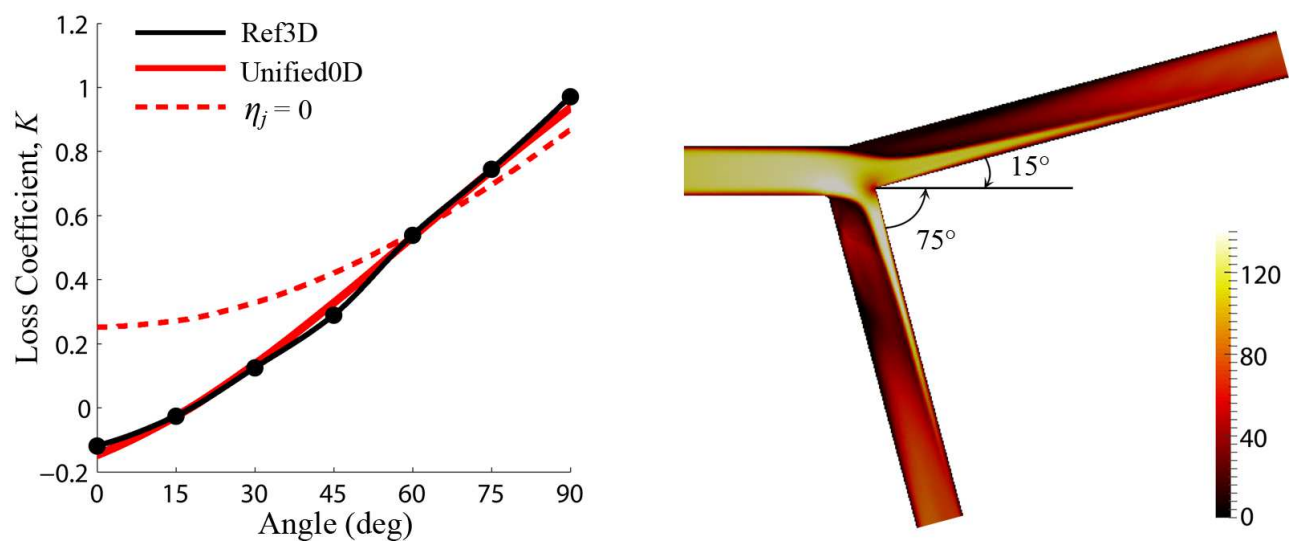

Figure 4. Loss coefficients $(K)$ for diverging flow in a Y-junction (Type 3 ) in which the angle between the outlets is fixed at 90 degrees but the orientation angle with respect to the inlet is varied between 0 and 90 degrees.

occurs between collectors, in particular from a collector with a large $\varphi$ (i.e. in which flow undergoes significant redirection) to a collector with a smaller $\varphi$ (i.e. lesser flow redirection).

The implications of this phenomenon for the reduced order formulation are assessed in Figure 4 for diverging flow in a bifurcation. In all cases, the angle between the two collectors is 90 degrees, but the orientation of this collector pair is varied between 0 and 90 degrees with respect to the inlet. Hence, when one collector is at 90 degrees, the other is at 0 degrees (thus forming a T-junction), whereas when one branch is at 45 degrees, so too is the other collector (thus forming a symmetric Y-junction). Figure 4 shows that the loss coefficient obtained from CFD (black line) progressively falls as collector angle $(\varphi)$ decreases, as would be expected, and even becomes negative below 15 degrees. Although equation (30) predicts the decreasing trend (red dashed line), these loss coefficients diverge from CFD results below 45 degrees, asymptoting to $>0.2$ at low angles and therefore not exhibiting the negative coefficients.

To empirically account for the energy exchange, the definition of pseudodatum area in equation (33) is modified as follows:

$$
A_{\mathrm{dat}, j}=\frac{q_{\mathrm{dat}}}{\left(1-\eta_{j}\right) u_{\mathrm{dat}}}
$$

noting that now pseudodatum area $\left(A_{\mathrm{dat}, j}\right)$ is collector-specific. The factor $\eta_{j}$ accounts for the energy exchange between collectors, with the denominator of (35) representing an effective pseudodatum velocity. $\eta_{j}$ was empirically determined on the basis of CFD results shown in Figure 4. To calculate $\eta_{j}$, it is convenient to reorient the junction so that the average of all collector angles is zero (i.e. mean $\left(\theta_{C}\right)=0$ ) and to ensure that $0 \leq \theta_{\text {dat }}<\pi$. Then, describing $\eta_{j}$ as the following linear function of $\theta_{\text {dat }}$ leads to satisfactory agreement with CFD results (compare solid black and red lines in Figure 4),

$$
\eta_{j}=\left[a_{0}\left(\pi-\theta_{\text {dat }}\right) \operatorname{sign}\left(\theta_{j}\right)+a_{1}\right]\left(1-\lambda_{j}\right)
$$

given coefficient values $a_{0}=0.8$ and $a_{1}=-0.2$. Note that the $\operatorname{sign}\left(\theta_{j}\right)$ term indicates whether flow passing into collector $j$ undergoes more (positive) or less (negative) redirection with respect to the average collector angle. The last term involving $\lambda_{j}$ is a weighting that enforces $\eta_{j}=0$ when $\lambda_{j}=1$, i.e. when there is only one collector and hence no opportunity for energy exchange between multiple collectors. Note that Eq. (36) is determined only from CFD data in Figure 4 and is applied henceforth without influence from CFD.

2.4.4. Implementation The Unified0D method for calculating loss coefficients was implemented in Matlab (R2014a, The MathWorks, Inc., Natick, MA, USA). The full code 'JunctionLossCoefficient.m' will be made publicly available on the Matlab Central website. An overview of the implementation is shown in Figure 5. We note that care must be taken to properly orient the branch 


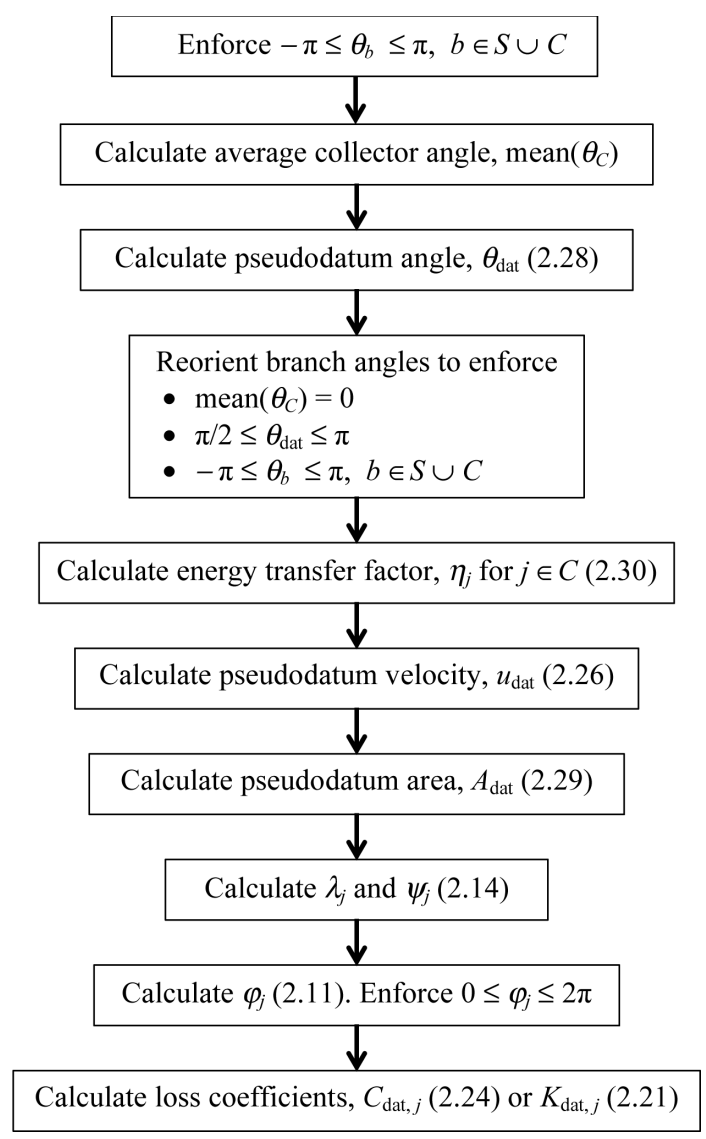

Figure 5. Flow chart outlining the procedure for calculating loss coefficients in any branch junction under arbitrary flow conditions.

angles to achieve results that are insensitive to differing angle conventions that may be chosen by a particular user. The code was incorporated into an existing 1D blood flow solver $(16 ; 44 ; 29)$ and was verified under both constant and pulsatile multi-directional flow conditions.

\section{RESULTS}

\subsection{Diverging Flow}

Figure 6 compares 'true' loss coefficients derived from CFD ('Ref3D') with those estimated via the unified 0D formulation ('Unified0D') for Type 1 flow (cf. Figure 1), with upper panels showing inlet to side branch coefficients and lower panels showing inlet to straight branch coefficients. Also shown are the loss coefficients obtained when assuming continuity of static pressure ('StaticP') or total pressure ('TotalP'). Note that, due to its wide use elsewhere in the literature, all results are presented in terms of the $K$ loss coefficient calculated via equation (8) rather than the $C$ coefficients calculated via equation (30).

Ref3D coefficients for Type 1 flow were relatively insensitive to $R e$ and were well estimated by Unified0D (Figure 6a,e), including negative coefficients for the straight branch. StaticP and TotalP underestimated side branch coefficients (TotalP worse) and overestimated straight branch coefficients (StaticP worse). Unified0D correctly predicted an increase in $K$ as flow fraction to the side branch increased (Figure $6 b, f$ ). Increasing side branch angle increased Ref3D $K$ for the side branch but made $K$ more negative in the straight branch, except for a large positive $K$ value at the largest side branch angle tested (Figure $6 c, g$ ). Decreasing side branch diameter caused 
(a)

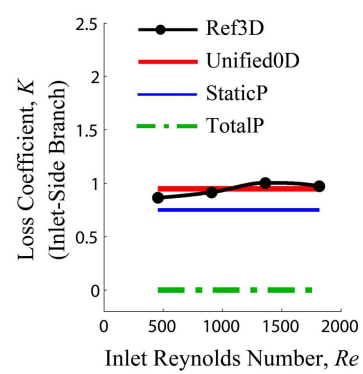

$(e)$

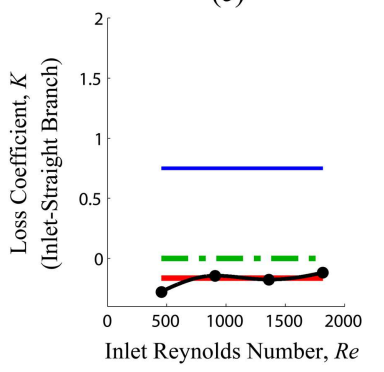

(b)

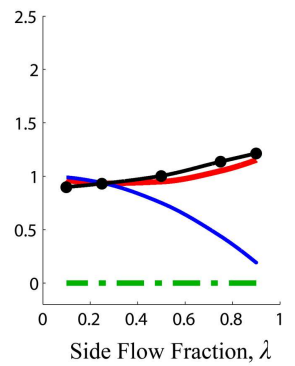

$(f)$
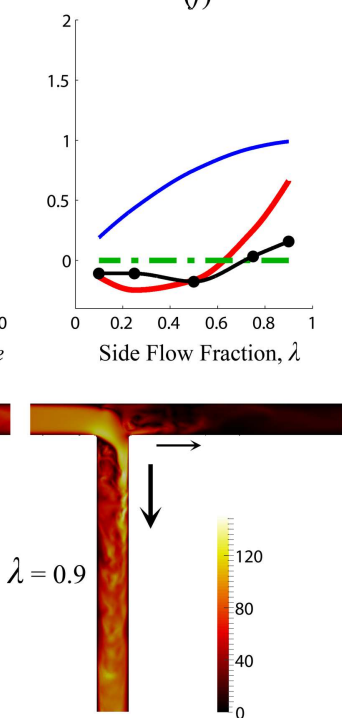

(c)

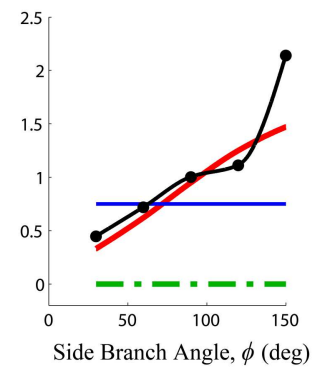

$(g)$
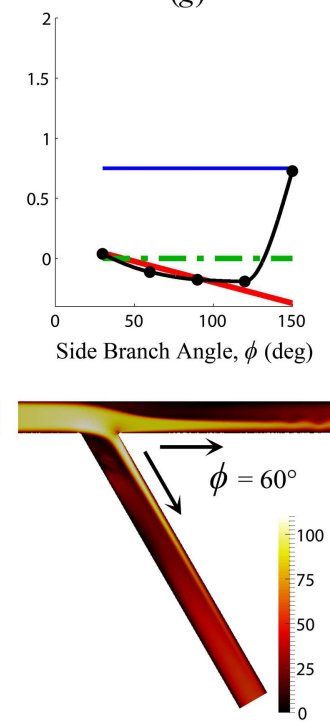

(d)

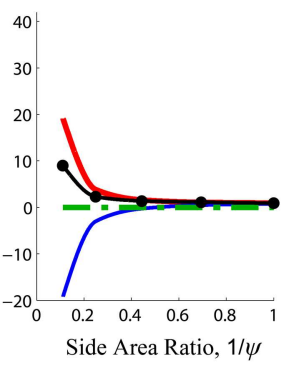

(h)
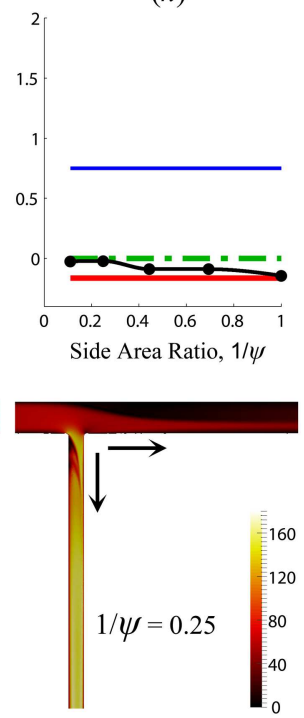

Figure 6. Loss coefficients $(K)$ for the inlet to side branch (upper panels) and inlet to straight branch (lower panels) for diverging flow in a T-junction (Type 1) with variation of $(a, e)$ Reynolds number, $R e$, $(b, f)$ side/straight branch flow ratio, $\lambda,(c, g)$ side branch angle, $\phi$, and $(d, h)$ side branch to main duct area ratio $(1 / \psi)$. Middle insets show the velocity fields from the 3D flow simulations corresponding to the data points indicated with arrows. Colour scales are in $\mathrm{cm} / \mathrm{s}$. Ref3D, Unified0D, StaticP and TotalP refer to losses calculated from 3D simulations (see Fig. 2), the unified reduced order pressure loss formulation and continuity of static and total pressure respectively. Inlet $R e$ is 1363 in $(b)$ and $(c)$, and 909 in $(d)$.

an exponential increase in $K$, noting that flow fraction to the side branch was fixed at 0.5 and hence velocity increased substantially as diameter decreased (Figure $6 d, h$ ). Overall, Unified0D predicted the main nature and magnitude of changes in $K$, whereas StaticP and TotalP substantially overestimated or underestimated $K$ and/or produced opposite trends to Ref3D (Figure $6 b, d$ ).

For Type 2 flow, there was a small increase in $K$ with $R e$, a greater dependence of $K$ on flow fraction and a still greater dependence on branch angle, but little dependence on the side branch (i.e. inlet) to main duct area ratio (Figure 7). Coefficients were generally well approximated by Unified0D, although changes with $R e$ were not predicted and $K$ was overestimated in the case of an outlet receiving a small flow fraction. TotalP incorrectly assumed $K=0$ while StaticP predicted no or incorrect trends in $K$ with flow fraction and branch angle, leading to large errors.

Ref3D coefficients for diverging flow in a Y junction (Type 3) displayed negligible dependence on $R e$, generally decreased with increasing flow fraction and increased with branching angle (Figure 8). A local maximum was found at a branch angle of 90 degrees, which is a special case of Type 3 flow that corresponds to Type 2 flow. Although Unified0D did not predict this feature, excellent general agreement was obtained in contrast to StaticP and TotalP. 
(a)

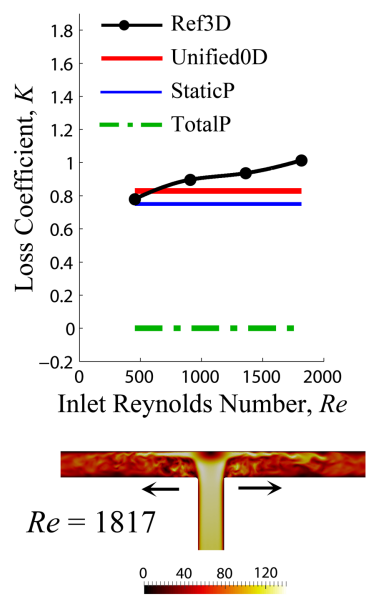

(b)
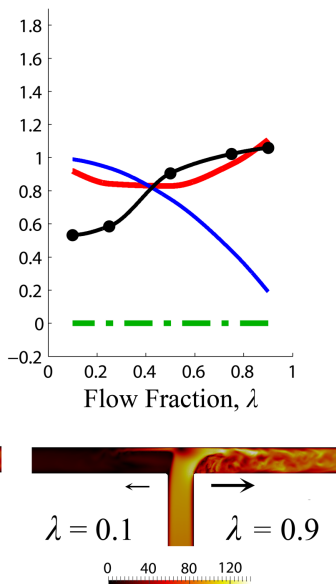

(c)
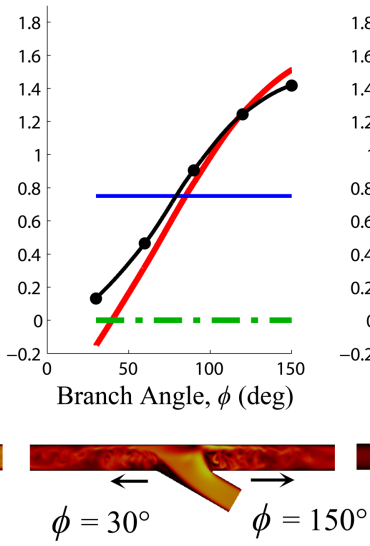

$\begin{array}{r}0 \quad 40 \quad 80 \quad 120 \\ \hline\end{array}$ (d)
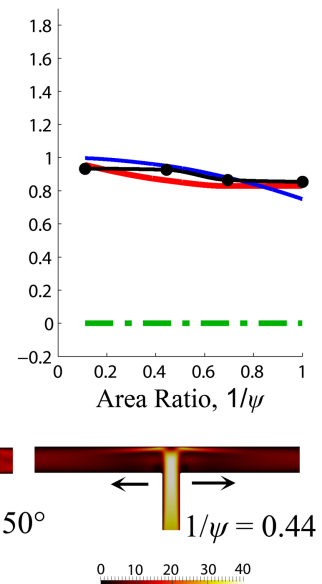

Figure 7. Loss coefficients $(K)$ for diverging flow from the side branch of a T-junction (Type 2) with variation of (a) Reynolds number, Re, $(b)$ outlet flow ratio, $\lambda,(c)$ branch angle, $\phi$, and $(d)$ inlet to outlet area ratio, $1 / \psi$. Inlet $R e$ is 1363 in $(b),(c)$ and $(d)$. Abbreviations as in Fig. 6.

(a)
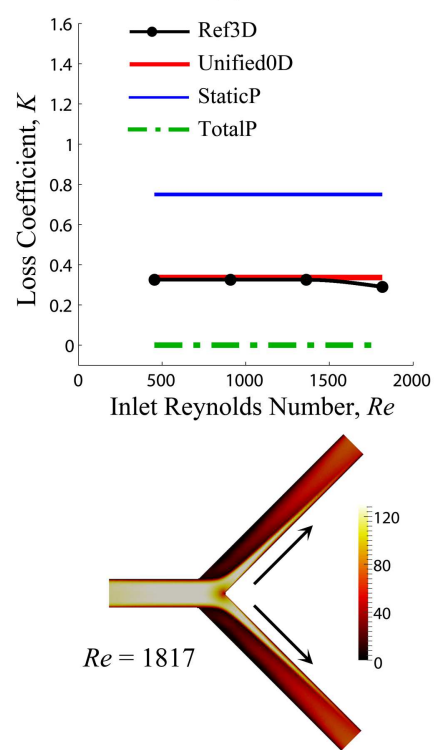

(b)
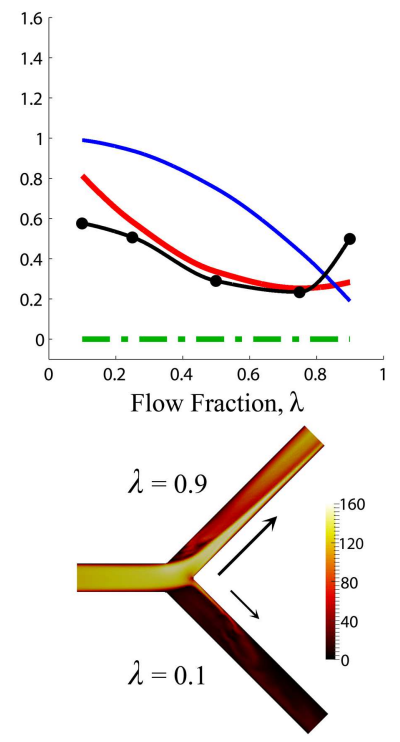

(c)

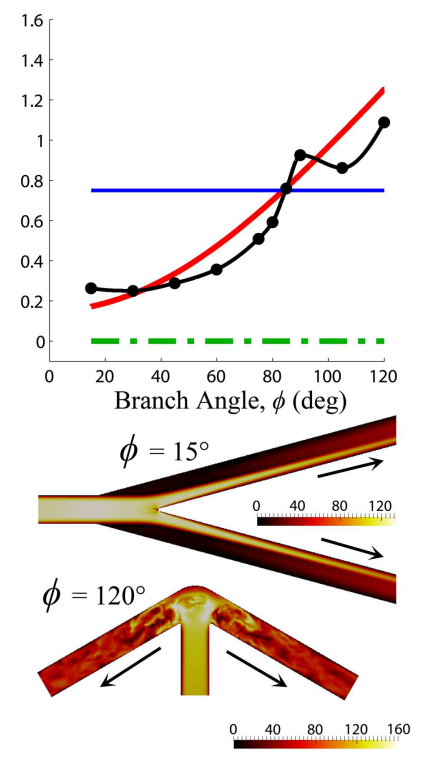

Figure 8. Loss coefficients $(K)$ for diverging flow in a Y-junction (Type 3 ) with variation of $(a)$ Reynolds number, $R e,(b)$ outlet flow fraction, $\lambda$, and $(c)$ branch angle, $\phi$. Inlet Re is 1817 in $(b)$ and $(c)$. Abbreviations as in Fig. 6.

\subsection{Converging Flow}

Ref3D coefficients for Type 4 flow showed negligible dependence on $R e$ (Figure 9). Increasing side branch flow fraction increased the side branch $K$ and substantially increased $K$ in the straight branch, noting that $K$ approaches -1 as side branch flow fraction approaches zero. Increasing side branch angle and decreasing side branch area ratio reduced $K$ for both inlets. Overall, Unified0D displayed excellent agreement with Ref3D, whereas StaticP and TotalP generally underestimated $K$ (StaticP being substantially worse than TotalP). Compared with Type 4 flow, Unified0D was less accurate for Type 5 flow (Figure 10), although Unified0D remained clearly superior to TotalP and StaticP. 
(a)

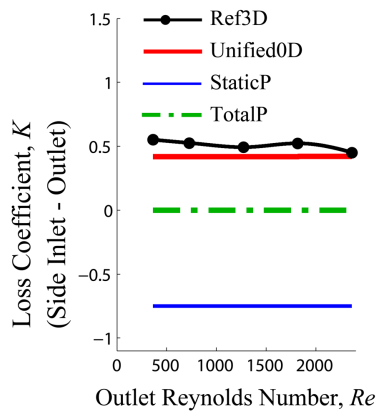

(e)

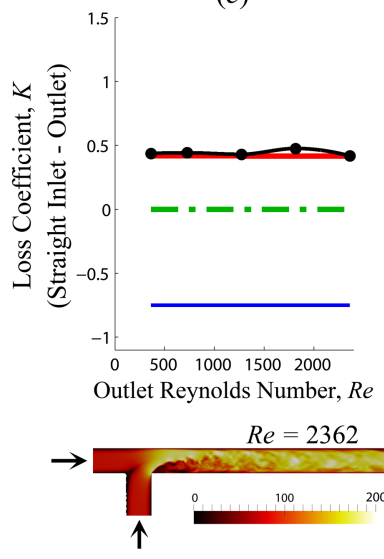

(b)

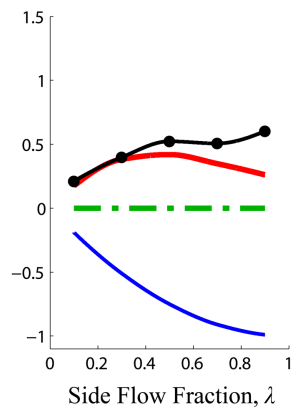

(f)
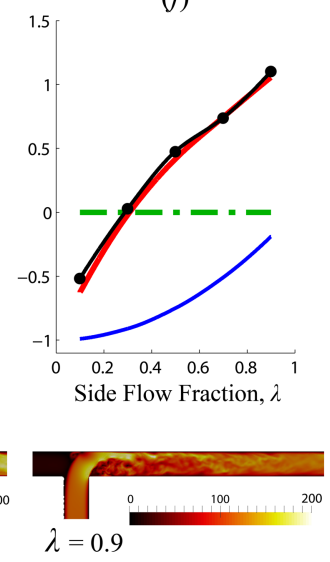

(c)

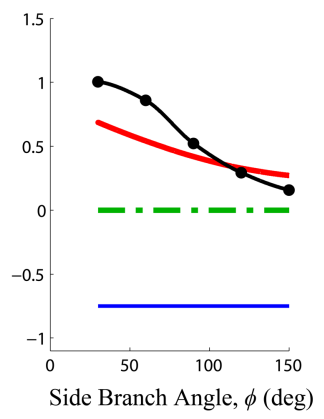

(g)
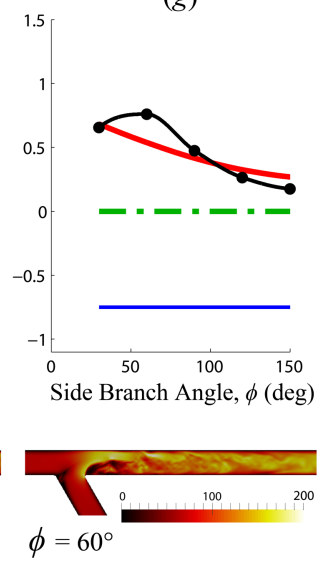

(d)

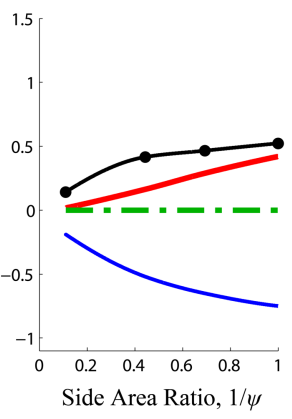

(h)

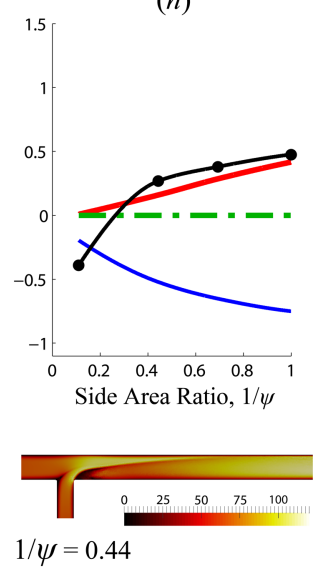

Figure 9. Loss coefficients $(K)$ for converging flow in a T-junction (Type 4) with variation of (a) Reynolds number, $R e(b)$ side/straight branch flow fraction, $\lambda,(c)$ side branch angle, $\phi,(d)$ side branch to main duct area ratio $1 / \psi$. Upper panels are coefficients for the side inlet, lower panels for the straight inlet. Outlet $R e$ is 1817 in $(b),(c)$ and $(d)$. Abbreviations as in Fig. 6.

(a)
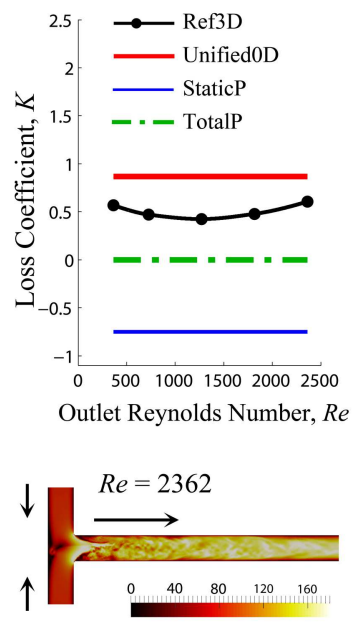

(b)
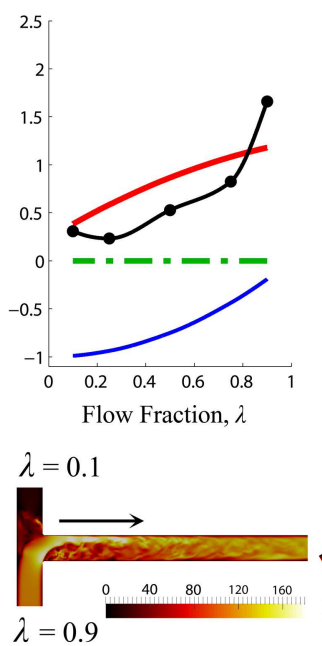

(c)
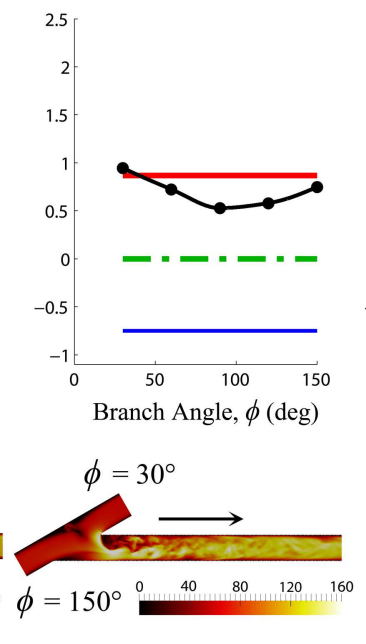

(d)

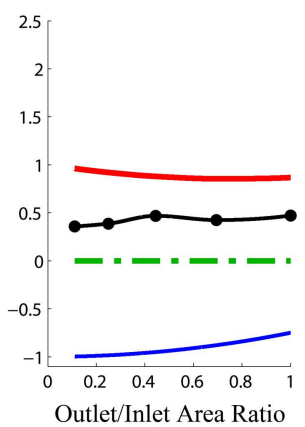

Area Ratio $=0.25$

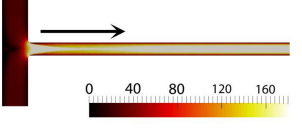

Figure 10. Loss coefficients $(K)$ for converging flow in a T-junction (Type 5) with variation of $(a)$ Reynolds number, $(b)$ side/straight branch flow ratio, $(c)$ side branch angle, $(d)$ side branch to main duct area ratio.

Outlet $R e$ is 1817 in $(b)$ and $(c)$. Re for both inlets in $(d)$ are fixed at 727. Abbreviations as in Fig. 6. 
(a)
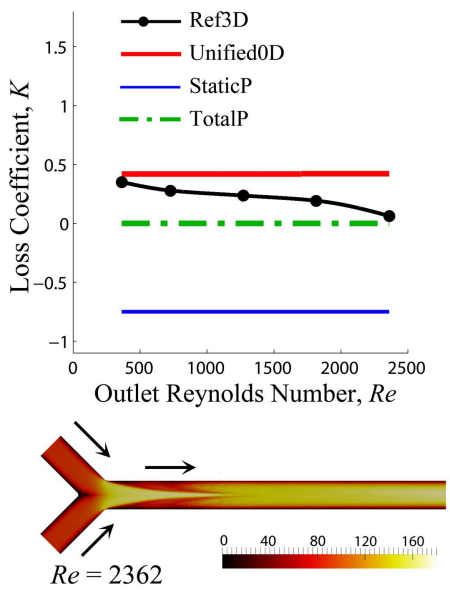

(b)

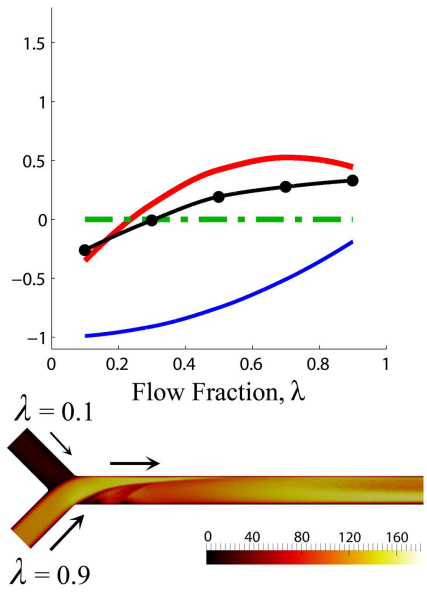

(c)

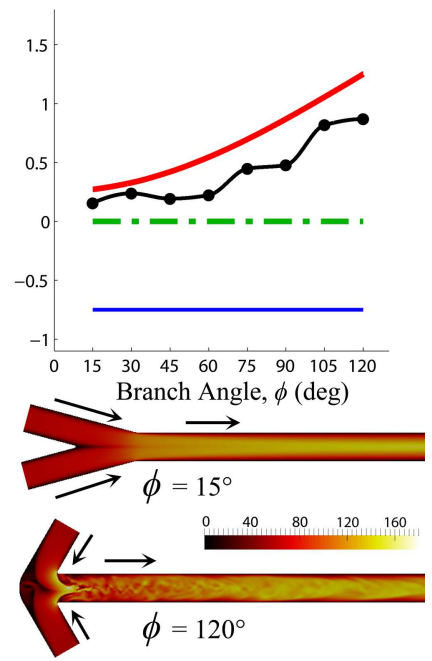

Figure 11. Loss coefficients $(K)$ for converging flow in a Y-junction (Type 3 ) with variation of $(a)$ Reynolds number, $(b)$ inlet flow ratio and $(c)$ branch angle. Outlet $R e$ is 1817 in $(b)$ and $(c)$. Abbreviations as in Fig.

6.

For converging flow in Y junctions (Type 6), $K$ (Ref3D) decreased from 0.35 to 0.15 as $R e$ was increased from 363 to 2362, a finding that was was not accounted for by any of the reduced order methods (Figure 11). Over the tested range of Re, flow fractions and branch angles, Unified0D tended to overestimate $K$, although it was generally more accurate than TotalP and was clearly superior to StaticP.

\subsection{Influence of inlet velocity profile}

All results presented thus far for Ref3D were generated with inlet velocity profiles described by equation 1 and $\gamma=9$, i.e. a mostly flat profile with a boundary layer. Figure 12 shows the impact of using $\gamma=2$ (a parabolic profile, long dashed lines) rather than $\gamma=9$ (Ref3D, solid black lines) on the loss coefficients over a range of $R e$. For Type 1 flow, a parabolic profile was associated with a $K$ increase of $\sim 0.25$ for the side branch, as compared with Ref3D, but a decrease of $\sim 0.35$ for the straight branch. In Type 2 flow, $K$ increased by 0.24 to 0.48 , depending on $R e$. For the remaining flow types (Type 3 to Type 6), velocity profile shape appeared to have a negligible influence on the loss coefficients.

\subsection{Influence of junction filleting}

In addition to inlet velocity profile, Figure 12 reveals the sensitivity of loss coefficients to the amount of filleting at the junction. Results labelled 'Ref3D' employed no filleting apart from a small amount of smoothing that was applied to avoid sharp corners. When filleting was incorporated (see top right inset of Figure 12), side branch $K$ decreased by $\sim 0.3$ for Type 1 flow, but had little effect for the straight branch $K$. Filleting decreased $K$ by $\sim 0.3$ for the side branch in Type 4 flow (and also for the straight branch, data not shown for clarity) and by $\sim 0.25$ for Type 5 flow. Filleting had a minor impact on $K$ for Type 2, Type 3 and Type 6 flows.

\subsection{Pulsatile flow}

Figure 13 shows results of a 1D model of pulsatile multidirectional flow in a T junction. Although not representing any specific in vivo situation, this model tests the limits of what is likely to be encountered in real applications. In particular, $R e$ ranged from approximately +2000 (supplier) to -2000 (collector) in each branch, while in the course of the simulation, junction flow underwent 

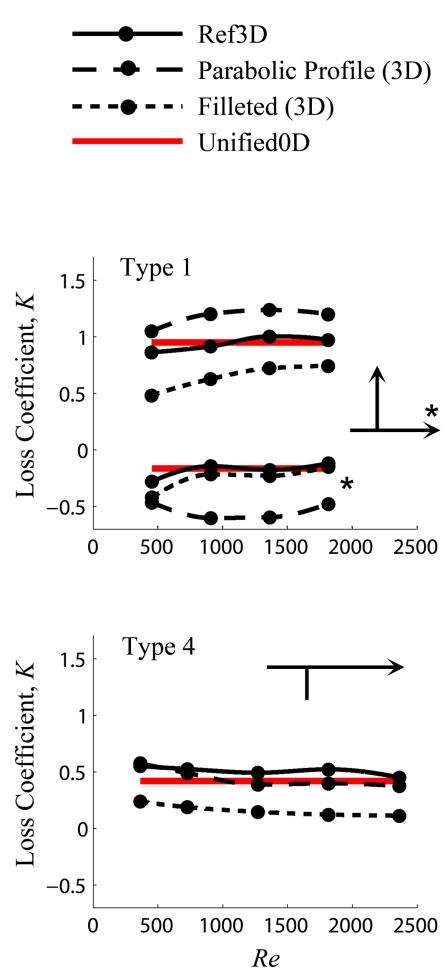
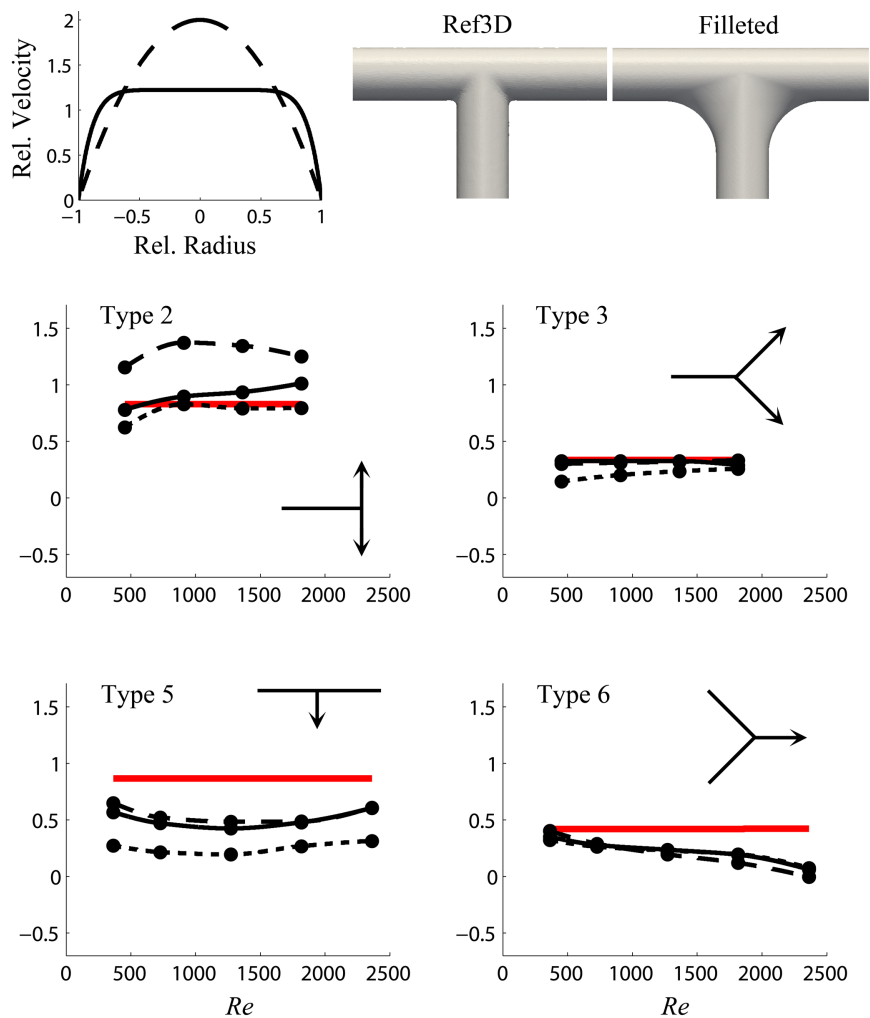

Figure 12. Influence of inlet velocity profile and filleting on junction loss coefficients $(K)$. Ref3D has no filleting and an inlet velocity profile described by equation (1) with $\gamma=9$. The parabolic velocity profile corresponds to $\gamma=2$. Coefficients produced by the Unified0D method are also displayed for reference. Note that for clarity, Type 4 straight branch coefficients are not shown, but are similar to the side branch coefficients.

all possible transitions between flow types pertinent to $\mathrm{T}$ junctions (Types 1, 2, 4 and 5). The Unified0D method produced smooth pressure and flow (or Re) waveforms and $C^{0}$ continuous transjunction pressure differences. By contrast, Bassett et al's method produced discontinuities in simulated velocity (Reynolds number) and pressure, as shown in the zoomed-in example in the lower panels of Figure 13. In this example, the discontinuity was associated with a pressure jump of $0.37 \mathrm{kPa}$ (or $2.8 \mathrm{mmHg}$ ).

\section{DISCUSSION}

Drawing from the work of Bassett et al (26), we proposed a unified technique for estimating pressure loss coefficients at tube or vascular junctions. The method is 'unified' in the sense that it can be applied to a junction of any number of branches of any size, joining at arbitrary angles and containing any flow regime. This contrasts with the conventional estimation of loss coefficients that has relied on relatively complicated empirical equations that are specific to particular junction configurations and flow regimes, an approach that has inherently limited applicability. A key benefit of the Unified0D method is that smooth solutions of pressure and flow can be obtained in reduced order models in which flow reversals lead to transient changes of flow regime (e.g. from converging to diverging). 

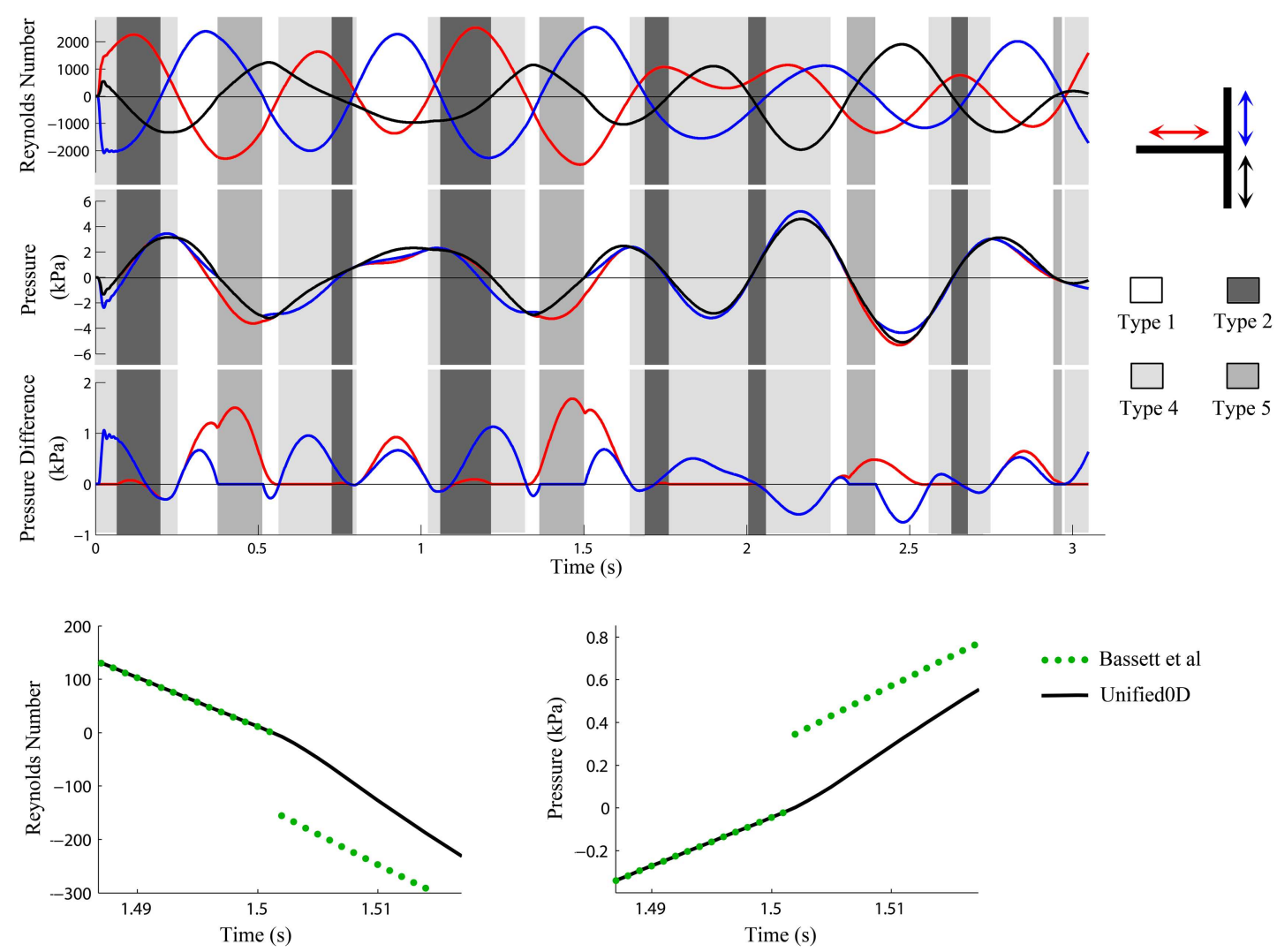

Figure 13. Time-varying Reynolds numbers, pressures and transjunction pressure differences in a onedimensional simulation of pulsatile multidirectional flow in a $\mathrm{T}$ junction with the Unified0D formulation (upper plots). Zoomed-in comparison of the solution obtained from Unified0D and Bassett et al's method (26), with the latter exhibiting discontinuities (lower plots).

\subsection{Validation with $C F D$}

The Unified0D method could be applied to an infinite number of junction/flow configurations, so we limited our validation study to junction types and flows that are most relevant to our field of interest, namely physiological flows. Reference loss coefficients were obtained from high resolution CFD spanning laminar and transitional test cases. Numerical convergence was determined on the basis of inlet-outlet pressure differences, as was appropriate for the purposes of this study. We note that assessment of convergence should be assessed on the basis of the quantity being studied, since convergence occurs at different rates for different quantities. For example, in a recent CFD challenge even normal/low resolution CFD was shown to predict similar pressure drops across a cerebral arterial stenosis compared with high resolution CFD (45). On the other hand, large differences have been demonstrated between normal and high resolution CFD when assessing local wall shear stress (WSS), oscillatory shear index (OSI) and kinetic energy ratio (KER) (46).

Overall, Unified0D displayed excellent agreement with CFD (Ref3D) over the physiological range of $R e$ and a wide range of flow fractions, branch angles and area ratios. Tests were presented in terms of six standard junction/flow types (Figure 1). Although empirical equations can potentially achieve near perfect agreement, given enough empirical constants, we consider the Unified0D approach to have achieved a satisfactory level of agreement while retaining the benefits of a simple and unified formulation, wide applicability and the ability to simulate reversing flows. Nevertheless, for applications requiring precise quantification of pressure losses and/or in situations where junction geometry is highly irregular, CFD, in vitro testing or direct measurement may remain unavoidable. 


\subsection{Influence of velocity profile and filleting}

As far as we are aware, this is the first study to investigate the effect of supplier velocity profile on junction loss coefficients. Our results suggested that loss coefficients were relatively insensitive to velocity profile for converging flow in $\mathrm{T}$ junctions and all $\mathrm{Y}$ junction flows. By contrast, loss coefficients in Type 1 and Type 2 flows (diverging $\mathrm{T}$ junction) displayed a clear dependence on supplier velocity profile (Figure 12). Compared with the mainly flat profile, a parabolic profile was associated with an increased $K$ (by approximately 0.5) in Type 2 flow and in the side branch of Type 1 flow, as might be expected given the higher peak velocity. Less expected was the finding that $K$ in the straight branch was lower in the case of a parabolic profile; this will be discussed further in the next section.

Filleting of the junction corners caused a minor to moderate reduction in $K$ for all flow types, as might be expected, except Type 6 where the effect was negligible. Although the Unified0D formulation could be adjusted to account for velocity profile and filleting effects, this would introduce significant additional complexity. Given the inherent uncertainties involved in reduced order flow modelling, the small errors introduced by ignoring these effects are not likely to be of great significance in most applications.

\subsection{Negative loss coefficients}

The meaning of negative junction loss coefficients has been discussed by a number of investigators $(47 ; 23 ; 48)$. A negative loss coefficient signifies that an exchange of energy has occurred between flow streams, noting that there must always be a net energy loss at a junction. As highlighted by Wood et al (23) and Liggett (48), use of the term 'loss coefficient' is therefore somewhat inaccurate, since its value is affected by energy exchange as well as energy loss. However, following Wood et al (23) we retain the term given its widespread use.

Previous discussion of negative loss coefficients has focused on converging flow in $\mathrm{T}$ junctions, where a low side branch flow fraction can lead to $K$ values as low as -1 . Wood et al (23) suggested that this occurs because "when a high-velocity stream combines with a low-velocity stream, a jet pump action takes place that transfers energy from the high-velocity stream to low-velocity stream".

As far as we are aware, negative loss coefficients have not been reported previously in diverging junction flows. We found slightly negative loss coefficients for the straight branch in diverging $\mathrm{T}$ junction flow (Type 1). These were affected by Reynolds number, flow fraction, side branch angle and side branch area ratio (Figure 6). Of particular interest was our finding that, for a fixed mean velocity but increased peak velocity (i.e. for a parabolic velocity profile compared with a mostly flat profile), $K$ increased for the side branch but decreased (i.e. became more negative and falling under $-0.5)$ for the straight branch (Figure 12).

A detailed analysis of the physics underlying negative loss coefficients in diverging flow is beyond the scope of this report. However, as with converging flow, we suggest that a form of energy exchange occurs between flow jets entering the two collector branches, modulated by peak velocity in the supplier branch. As initial support for this, we found that doubling $\eta$ in equation 35 closely reproduced the opposing effects of velocity profile on Type 1 loss coefficients shown in Figure 12 for Ref3D.

\subsection{Relevance to reduced order physiological flow modelling}

Existing reduced order models of physiological flow have almost universally enforced continuity of static or total pressure (StaticP or TotalP) at vascular junctions. Results of the current study suggest that neither of these methods reliably estimate $K$ loss coefficients, with typical errors of up to 1.5 , and neither method could be judged as generally superior to the other. Nevertheless, this does not imply that use of StaticP or TotalP will always lead to significant inaccuracies in simulated pressure and flow. Whether errors are physiologically significant will depend on the magnitude of dynamic pressure. In adult systemic arteries, for example, static pressure is typically in the order of $13 \mathrm{kPa}$, compared with only $0.5 \mathrm{kPa}$ for dynamic pressure; hence errors of less than $1 \mathrm{mmHg}$ ( $\sim 1 \%$ of arterial pressure) would be expected, although errors may accumulate where multiple 

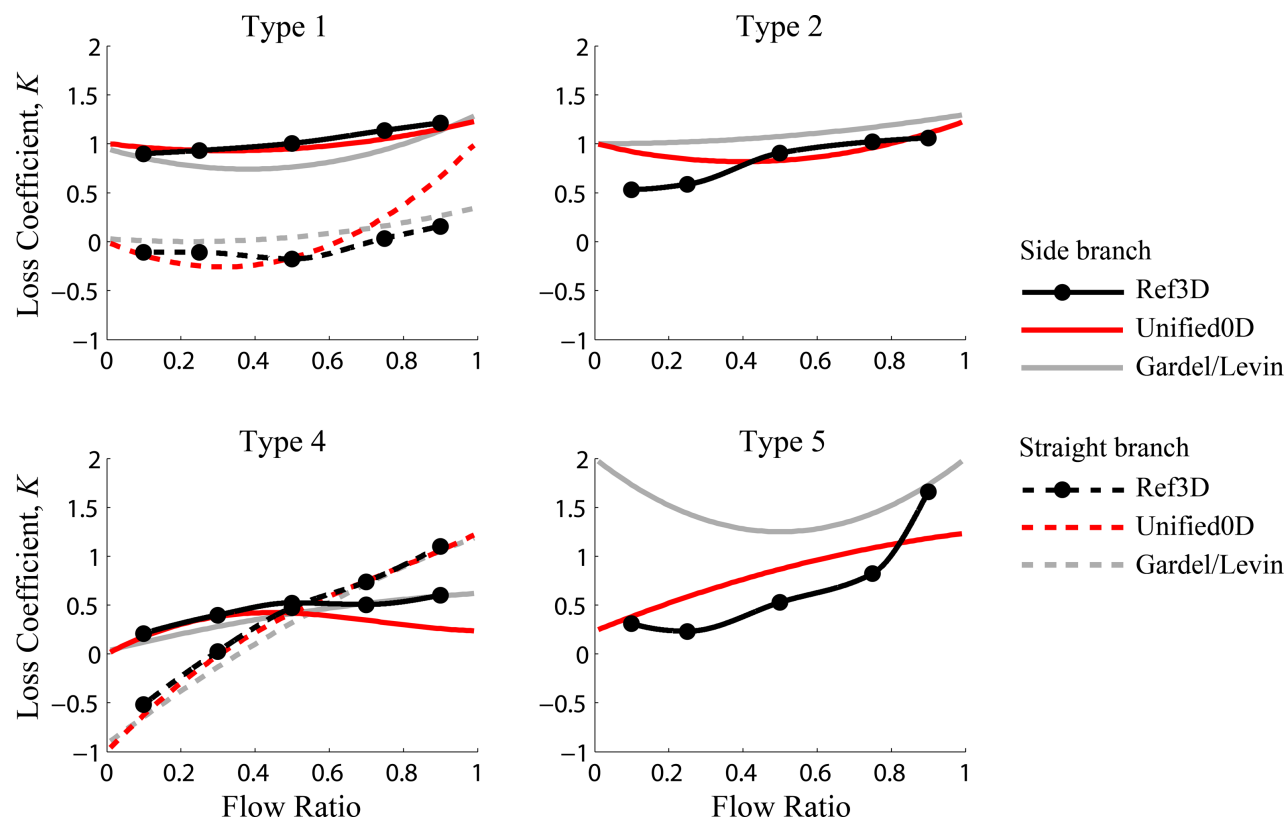

Straight branch

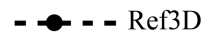

- - - - - Unified0D

$-m=-$ Gardel/Levin

Figure 14. Comparison of $K$ loss coefficients derived from computational fluid dynamics (Ref3D) for $R e$ of 1363 (Type 1,2) and 1817 (Type 4,5), the proposed reduced order formulation (Unified0D), and the empirical equations described by A. Gardel and S.R. Levin (as republished in English by Wood et al (23)) and validated at $R e>10^{5}$.

branching generations are simulated. As an example, taking $K=0.35$, which is typical of diverging or converging flow in a $\mathrm{Y}$ junction, and a maximum velocity of $50 \mathrm{~cm} / \mathrm{s}$, the expected pressure loss is approximately $1 \mathrm{mmHg}$ after 3 bifurcations. For a maximum velocity of $200 \mathrm{~cm} / \mathrm{s}$, which occurs in normal young adults during exercise (49), the loss could reach almost $17 \mathrm{mmHg}$ after 3 bifurcations, which is quite significant. In systemic veins, although static pressure may approach zero (50), dynamic pressure typically reaches no more than $\sim 100 \mathrm{~Pa}$ (i.e. approximately $1 / 10$ $\mathrm{mmHg}$ ); which is unlikely to be of physiological significance. However, exercise conditions may again make the losses more significant.

Accurately accounting for pressure losses may be essential in some physiological flow models, e.g. when considering abnormal (pathological or surgically-induced) arterial configurations (20). In the fetal ductus arteriosus, dynamic pressure may reach $1.4 \mathrm{kPa}(\sim 10 \mathrm{mmHg})$ under normal conditions or $2.6 \mathrm{kPa}(\sim 19 \mathrm{mmHg})$ in cases of ductal constriction, compared with a static pressure of $\sim 6.7 \mathrm{kPa}(51)$. Pulmonary arterial and venous networks consist of many generations of junctions, with normal dynamic pressures reaching $0.4 \mathrm{kPa}$ (arteries and veins) compared with static pressures in the order of $1.5 \mathrm{kPa}$ (arteries) and $0.9 \mathrm{kPa}$ (veins) $(52 ; 50)$. In the airways, which also contain many junctions, dynamic pressure ranges between $0.6 \mathrm{kPa}$ at rest and $60 \mathrm{kPa}$ during intensive exercise (53), while normal and maximal peak inspiratory (static) pressures are in the order of $2.5 \mathrm{kPa}$ and $10 \mathrm{kPa}$ respectively. The Unified0D method will enable efficient estimation of junction pressure losses in these and other applications, with substantially greater accuracy than StaticP or TotalP methods. Our method is particularly relevant for situations in which significant flow reversals occur, such as in the ductus arteriosus soon after birth (54), in the context of aortic or pulmonary regurgitation (55), or ventriculo-coronary fistulae (35).

\subsection{General applicability of the Unified0D method}

4.5.1. High Reynolds numbers The applicability of the Unified0D method is by no means limited to the study of physiological flows. While our validation study only covered $R e$ in the range 350 to 2400 , the loss coefficients obtained were similar to those reported previously for high Reynolds number flows $\left(R e>10^{5}\right)$, as described with the empirical equations of Gardel and Levin (23) and 
plotted in Figure 14. Indeed, the Unified0D method is an updated version of the method described by Bassett et al (26), which was validated in the context of turbulent flow. Along with the relative insensitivity of loss coefficients to Reynolds number, it is not surprising that the Unified0D method is likely to be valid not only for laminar and transitional flows, but fully turbulent flow also.

4.5.2. Low Reynolds numbers The majority of work relating to junction loss coefficients has been devoted to high $R e$ flow, but a few investigators have studied junction losses at low $R e$, which may be of interest in micro-scale flow devices. Kiser et al (56) reported results of a CFD study that suggested $K \sim 1$ for $200<R e<2000$ (in agreement with our results). However, for $R e<200$ an inverse relationship with $R e$ was found, with $K$ reaching 300-400 at $R e=1$. A similar trend but coefficients at least one order of magnitude greater were reported by Jamison and Villemonte (57) on the basis of tube experiments. Kiser et al (56) suggested this large quantitative discrepancy may have been due to differences in the junction geometries employed.

However, we suspect that the large coefficients at low Re reported in both of these studies may be spurious. We also performed a CFD simulation of diverging flow in a 45 degree Y junction (as in Figure 8) for $R e=1$ and found that the associated loss coefficient was exquisitely sensitive to how the pressure difference $\left(\Delta p_{i, j}\right)$ was measured. Using the method shown in Figure 2, we obtain a loss coefficient of almost $K=-5$. Kiser et al (56) did not extrapolate pressure to $x=0$ as we did, but calculated $\Delta p_{i, j}$ as the difference between pressures at the inlet and outlet of the junction (i.e. at approximately $x= \pm \mathrm{D}$ ). Using this method, we obtain $K=91$, which is close to the values reported by Kiser et al (56). On the other hand, if we take particular care to extrapolate pressure to the exact point where the incoming velocity stream starts to expand and divide (in our model, at $x=-D / 6$ ), we obtain $K=0.4$, which is very similar to the values obtained at $R e>350$ (see Figure $8 a$ ) $^{\ddagger}$. We conclude that the large loss coefficients reported previously likely arose from imprecise calculations of reference $\Delta p_{i, j}$, by excluding a small amount of Poiseuille resistance in the junction region. These observations call into question the purported $R e$-dependence of loss coefficients at low $R e$, and suggest that the Unified0D method is also valid in the low $R e$ range.

\subsection{Flow development and the calculation of junction pressure differences}

In this paper, $\Delta p_{i, j}$ was calculated by linearly extrapolating near-junction pressure to the junction centre $(x=0)$, as described by Gardel (22) and shown in Figures 2 and 15a. It should be noted, however, that this method ignores additional losses that occur downstream as flow develops. At the range of $R e$ tested in this study, such losses occur over approximately 20-60 diameters. An alternative method for calculating $\Delta p_{i, j}$ that incorporates these losses is shown in Figure 15b (58). Noting that pressure invariably developed in an approximately exponential fashion, an exponential curve was fit to the pressure profile. The point at which the spatial pressure gradient fell to the value predicted for Poiseuille flow was then identified (i.e. $\left.d p / d x=8 \mu u / R^{2}\right)^{\S}$. A line with this slope was then extrapolated to $x=0$, resulting in an alternative $\Delta p_{i, j}$ that incorporates both near-junction and developing flow related losses. In the remainder of this section, results pertaining to this alternative estimate of $\Delta p_{i, j}$ are labelled 'far', while those obtained from the method in Figure 2 and $15 a$ are labelled 'near'.

Analysis of all data presented in Figures 6 to 11 revealed a relatively predictable relationship between $\Delta p_{i, j}$ (near) and $\Delta p_{i, j}$ (far), along with associated loss coefficients. In particular, we found that the following relations yielded acceptable agreement between Ref3D(far) and Unified0D(far) loss coefficients,

$$
K_{j, \text { far }}=K_{j, \text { near }}+0.00035 R e
$$

for all diverging flows, and

$$
K_{j, \text { far }}=K_{j, \text { near }}+0.8
$$

\footnotetext{
¥For $R e>350$, loss coefficients differed by less than 0.1 if pressures were extrapolated to the point where the velocity stream began to diverge, instead of to $x=0$ as in Figure 2.

$\S$ In some cases, this point lay up to several diameters outside the computational domain
} 
for all converging flows. The adequacy of these relations is reflected in Figure 15d, which reveals an $R^{2}$ of 0.89 when comparing Ref3D(far) and Unified0D(far). This compares with $R^{2}=0.66$ for Ref3D(near) vs. Unified0D(near). Importantly, we found that the extra losses related to flow development were predominantly influenced by perturbations at the junction, with little 'memory' of the inlet velocity profile being transmitted across the junction.

While $K_{j \text {,far }}$ can therefore be estimated reliably via a slight adjustment to the Unified0D method, we suggest that use of $K_{j \text {,near }}$ may be more desirable in 1D physiological flow simulations. Specifically, in such simulations the evolution of pressure and flow in the axial dimension is sought. Using $K_{j \text {,far }}$ would lump losses that may occur over a large distance to a single point (the junction). A preferable approach would be to tune the viscous term in the 1D segments to account for extra losses that occur due to developing flow. It is already common practice to use $\gamma=9$ (a mostly blunt velocity profile) rather than $\gamma=2$ (parabolic profile) in 1D blood flow models, based on measured velocity profiles $(27 ; 28 ; 3 ; 29)$. This may approximately account for the developing flow losses, along with the fact that the velocity profile is generally not parabolic in fully-developed pulsatile flows. However, this issue may warrant further investigation.

\subsection{Limitations}

There were several limitations to this study. Although the Unified0D method can be applied to junctions of any number of branches, validation with CFD was limited to three-branch junctions.
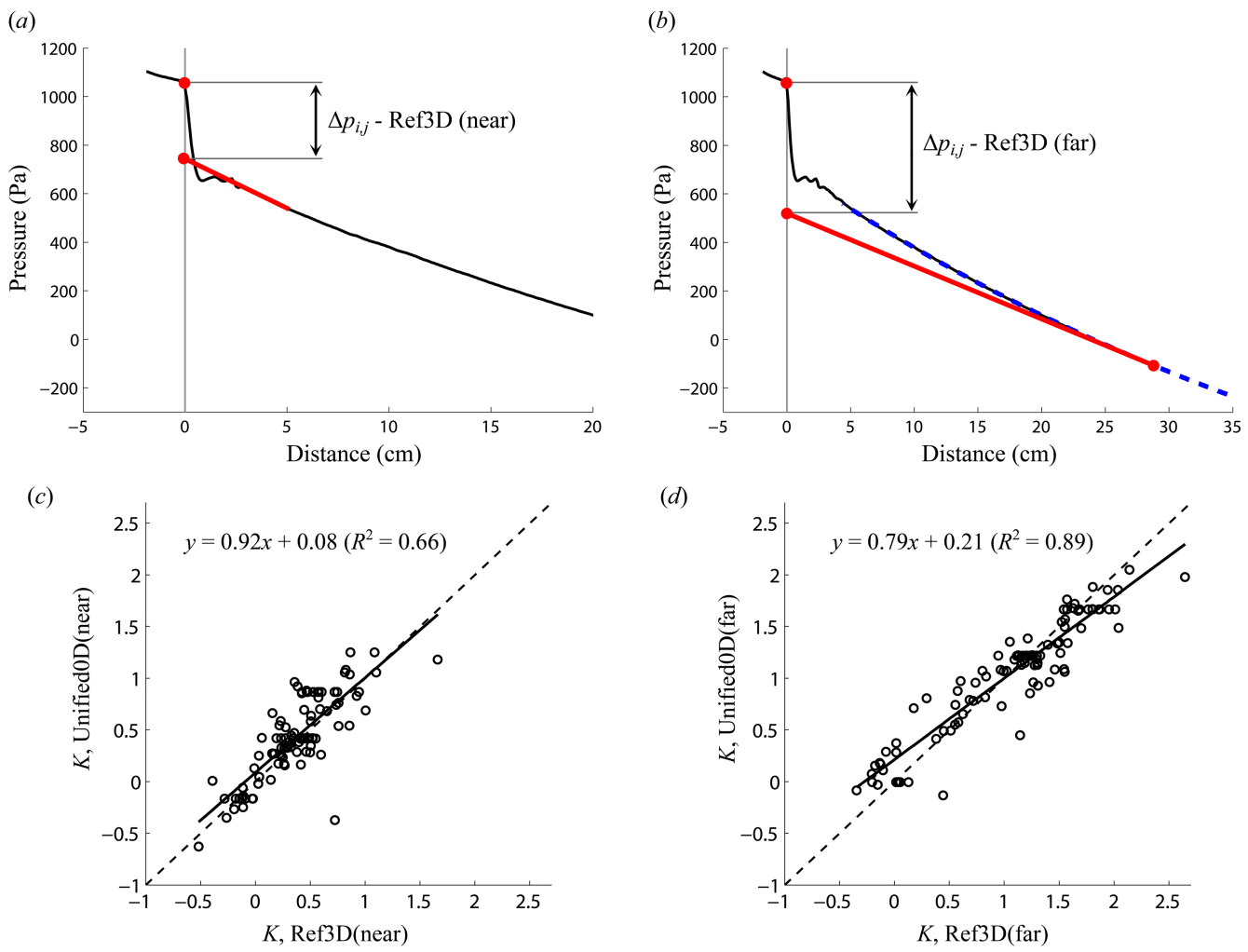

Figure 15. Pressure differences between branch $i$ and $j\left(\Delta p_{i, j}\right)$ arising from junction flow perturbations may be calculated by linearly extrapolating the nearby pressure to the junction origin, as in Figure 2( $a$, 'near'), or by extrapolating the Poiseuille pressure gradient from the point where flow become fully developed $(b$, 'far'). Black lines are cross-sectionally averaged pressures, red lines show the extrapolation to $x=0$ (the junction centre). In $(b)$, the blue dashed line is an exponential fit of the pressure, while the blue circle at $x \approx 29$ indicates the point where flow becomes fully-developed. (c) Linear regression of Ref3D(near) vs. Unified0D(near) loss coefficients, and (d) Ref3D(far) vs. Unified0D(far) coefficients, where Unified0D(far) coefficients are calculated via equations (37) and (38). 
We also did not fill the entire parameter space for three-branch junctions, choosing instead to vary only one parameter (Re, flow fraction, branch angle, area ratio) at a time. We did not perform validation under fully turbulent conditions, although features of transitional flow were evident at the highest $R e$ tested. The agreement between Ref3D and Unified0D for all possible three-branch junction flows has therefore not been established, however doing so would require a large number of additional simulations. All validation tests were performed with the full 3D transient NavierStokes equations, but under constant inflow conditions; therefore we have not validated the method for pulsatile inflows. Applying the Unified0D method to pulsatile flow assumes that pressure loss at a given instant is equivalent to the loss under steady flow conditions. Bassett et al (26) found that this assumption held under unsteady flows and even flows containing shock waves. The quasisteady flow assumption was also supported by data of Benson et al (41) and Bingham and Blair (42). Nevertheless, in future studies we plan to evaluate the Unified0D method under pulsatile flow conditions with high fidelity in vivo pressure recordings.

\section{CONCLUSION}

A unified reduced-order (Unified0D) method has been described to estimate pressure losses at branch junctions. By defining a pseudodatum branch, the method can be applied to any junction configuration and any flow regime. The method was validated against high resolution CFD for a wide range parameters and all possible flow types in three-branch junctions. Although a physiological range of $R e$ was considered, we have argued that the method appears to be valid at any $R e$, implying potential for broad applicability. When incorporated into reduced-order flow simulations, smooth pressure/flow waveforms are obtained where flow reversals cause the junction flow type to change abruptly. For applications in which junction pressure losses are non-negligible, substantially greater accuracy is achieved by applying the Unified0D method, compared with enforcing continuity of static or total pressure.

\section{REFERENCES}

[1] Matthys KS, Alastruey J, Peiró J, Khir AW, Segers P, Verdonck PR, Parker KH, Sherwin SJ. Pulse wave propagation in a model human arterial network: Assessment of 1-D numerical simulations against in vitro measurements. J Biomech 2007; 40(15):3476-3486.

[2] Reymond P, Merenda F, Perren F, Rufenacht D, Stergiopulos N. Validation of a onedimensional model of the systemic arterial tree. Am J Physiol Heart Circ Physiol 2009; 297(1):H208-222.

[3] Alastruey J, Nagel SR, Nier BA, Hunt AAE, Weinberg PD, Peiró J. Modelling pulse wave propagation in the rabbit systemic circulation to assess the effects of altered nitric oxide synthesis. J Biomech 2009; 42(13):2116-2123.

[4] Mynard JP, Penny DJ, Smolich JJ. Scalability and in vivo validation of a multiscale numerical model of the left coronary circulation. Am J Physiol Heart Circ Physiol 2014; 306(4):H517H528.

[5] Westerhof N, Bosman F, De Vries CJ, Noordergraaf A. Analog studies of the human systemic arterial tree. J Biomech 1969; 2(2):121-134.

[6] Lanzarone E, Liani P, Baselli G, Costantino ML. Model of arterial tree and peripheral control for the study of physiological and assisted circulation. Med Eng Phys 2007; 29(5):542-555.

[7] Broome M, Maksuti E, Bjallmark A, Frenckner B, Janerot-Sjoberg B. Closed-loop realtime simulation model of hemodynamics and oxygen transport in the cardiovascular system. Biomed Eng Online 2013; 12(1):69. 
[8] Pollack GH, Reddy RV, Noordergraaf A. Input impedance, wave travel, and reflections in the human pulmonary arterial tree: Studies using an electrical analog. IEEE Trans Biomed Eng 1968; 15(3):151-64.

[9] Olufsen MS. Structured tree outflow condition for blood flow in larger systemic arteries. Am J Physiol Heart Circ Physiol 1999; 276(1):H257-268.

[10] Burrowes KS, Hunter PJ, Tawhai MH. Anatomically based finite element models of the human pulmonary arterial and venous trees including supernumerary vessels. J Appl Physiol 2005; 99(2):731-738.

[11] Qureshi MU, Vaughan GA, Sainsbury C, Johnson M, Peskin C, Olufsen M, Hill NA. Numerical simulation of blood flow and pressure drop in the pulmonary arterial and venous circulation. Biomech Model Mechanobiol 2014; 13(5):1137-1154.

[12] Ma B, Lutchen KR. An anatomically based hybrid computational model of the human lung and its application to low frequency oscillatory mechanics. Ann Biomed Eng 2006; 34(11):16911704.

[13] Clavica F. Computational and experimental time domain, one dimensional models of air wave propagation in human airways. PhD Thesis, Brunel University 2012.

[14] Ismail M, Gravemeier V, Comerford A, Wall WA. A stable approach for coupling multidimensional cardiovascular and pulmonary networks based on a novel pressure-flow rate or pressure-only neumann boundary condition formulation. Int J Numer Methods Biomed Eng 2014; 30(4):447-469.

[15] Sherwin SJ, Formaggia L, Peiró J, Franke V. Computational modelling of 1D blood flow with variable mechanical properties and its application to the simulation of wave propagation in the human arterial system. Int J Num Meth Fluids 2003; 43(6-7):673-700.

[16] Mynard JP, Nithiarasu P. A 1D arterial blood flow model incorporating ventricular pressure, aortic valve and regional coronary flow using the locally conservative Galerkin (LCG) method. Comm Numer Methods Eng 2008; 24(5):367-417.

[17] Fullana JM, Zaleski S. A branched one-dimensional model of vessel networks. J Fluid Mech 2009; 621(1):183-204.

[18] Marchandise E, Willemet M, Lacroix V. A numerical hemodynamic tool for predictive vascular surgery. Med Eng Phys 2009; 31(1):131-144.

[19] Stergiopulos N, Young DF, Rogge TR. Computer simulation of arterial flow with applications to arterial and aortic stenoses. J Biomech 1992; 25(12):1477-1488.

[20] Steele BN, Jing W, Ku JP, Hughes TJR, Taylor CA. In vivo validation of a one-dimensional finite-element method for predicting blood flow in cardiovascular bypass grafts. IEEE Trans Biomed Eng 2003; 50(6):649-656.

[21] Huberts W, Bode AS, Kroon W, Planken RN, Tordoir JHM, van de Vosse FN, Bosboom EMH. A pulse wave propagation model to support decision-making in vascular access planning in the clinic. Med Eng Phys 2012; 34(2):233-248.

[22] Gardel A. Les pertes de charge dans les écoulements au traverse de branchments en té. Bull Technique de la Suisse Romande 1957; 83(9):123-130.

[23] Wood DJ, Reddy LS, Funk JE. Modeling pipe networks dominated by junctions. J Hydraul Eng 1993; 119(8):949-958.

[24] San O, Staples AE. An improved model for reduced-order physiological fluid flows. Journal of Mechanics in Medicine and Biology 2012; 12(03). 
[25] Taylor CA, Draney MT. Experimental and computational methods in cardiovascular fluid mechanics. Annu. Rev. Fluid Mech. 2004; 36:197-231.

[26] Bassett MD, Pearson RJ, Fleming NP, Winterbone DE. A multi-pipe junction model for onedimensional gas-dynamic simulations. SAE Trans 2003; 112(3):565-583.

[27] Smith NP, Pullan AJ, Hunter PJ. An anatomically based model of transient coronary blood flow in the heart. SIAM J Appl Math 2002; 62(3):990-1018.

[28] Čanić S, Kim EH. Mathematical analysis of the quasilinear effects in a hyperbolic model blood flow through compliant axi-symmetric vessels. Math Meth Appl Sci 2003; 26(14):1161-1186.

[29] Mynard JP, Penny DJ, Davidson MR, Smolich JJ. The reservoir-wave paradigm introduces error into arterial wave analysis: A computer modelling and in-vivo study. J Hypertens 2012; 30(4):734-743.

[30] Logg A, Mardal K, Wells G. Automated solution of differential equations by the finite element method, vol. 84. Springer, 2012.

[31] Mortensen M, Valen-Sendstad K. Oasis - a high-level/high-performance open source navierstokes solver. Computer Physics Communications 2014; In Press.

[32] Goda K. A multistep technique with implicit difference schemes for calculating two-or threedimensional cavity flows. J Comput Phys 1979; 30(1):76-95.

[33] Simo JC, Armero F. Unconditional stability and long-term behavior of transient algorithms for the incompressible navier-stokes and euler equations. Comput Meth Appl Mech Eng 1994; 111(1-2):111-154.

[34] Barnard ACL, Hunt WA, Timlake WP, Varley E. A theory of fluid flow in compliant tubes. Biophys J 1966; 6(6):717-724.

[35] Mynard JP, Davidson MR, Penny DJ, Smolich JJ. A numerical model of neonatal pulmonary atresia with intact ventricular septum and rv-dependent coronary flow. Int J Numer Methods Biomed Eng 2010; 26(7):843-861.

[36] Stettler J, Niederer P, Anliker M. Theoretical analysis of arterial hemodynamics including the influence of bifurcations: Part i. Ann Biomed Eng 1981; 9(2):145-164.

[37] Formaggia L, Lamponi D, Quarteroni A. One-dimensional models for blood flow in arteries. J Eng Math 2003; 47:251-276.

[38] Olufsen MS, Peskin CS, Kim WY, Pedersen EM, Nadim A, Larsen J. Numerical simulation and experimental validation of blood flow in arteries with structured-tree outflow conditions. Ann Biomed Eng 2000; 28:1281-1299.

[39] Sherwin SJ, Franke V, Peiró J, Parker K. One-dimensional modelling of a vascular network in space-time variables. J Eng Math 2003; 47(3):217-250.

[40] Gardel A. Les pertes de charge dans les écoulements au travers de branchments en té. Bull Technique de la Suisse Romande 1957; 83(10):143-148.

[41] Benson RS, Woollatt D, Woods WA. Unsteady flow in simple branch systems. Proc Inst Mech Eng 1963-64; 178(Pt 31(iii)):24-49.

[42] Bingham JF, Blair GP. An improved branched pipe model for multi-cylinder automotive engine calculations. Proc Inst Mech Eng 1985; 199(D1):65-77.

[43] Hager WH. An approximate treatment of flow in branches and bends. Proc Inst Mech Eng 1984; 198C(4):63-69. 
[44] Mynard JP, Davidson MR, Penny DJ, Smolich JJ. Robustness of the p-u and lnd-u loop wave speed estimation methods: Effects of the diastolic pressure decay and vessel wall nonlinearities. Proceedings of the 33rd Annual International Conference of the IEEE Engineering in Medicine and Biology Society, Boston, MA, USA, 2011; 6446-6449.

[45] Steinman DA, Hoi Y, Fahy P, Morris L, Walsh MT, Aristokleous N, Anayiotos AS, Papaharilaou Y, Arzani A, Shadden SC, et al.. Variability of computational fluid dynamics solutions for pressure and flow in a giant aneurysm: The asme 2012 summer bioengineering conference cfd challenge. J Biomech Eng 2013; 135(2):021 016.

[46] Valen-Sendstad K, Steinman DA. Mind the gap: Impact of computational fluid dynamics solution strategy on prediction of intracranial aneurysm hemodynamics and rupture status indicators. American Journal of Neuroradiology 2013; .

[47] Blaisdell FW, Manson PW. Loss of energy at sharp-edged pipe junctions in water conveyance systems, vol. 1281. Agricultural Research Service, US Department of Agriculture, 1963.

[48] Liggett JA. Discussion of "modeling pipe networks dominated by junctions" by don j. wood, 1 . srinivasa reddy, and je funk (august, 1993, vol. 119, no. 8). J Hydraul Eng 1994; 120(12):14861489.

[49] Salmasi AM, Doré C. Variation of aortic blood velocity with age at rest and during exercise in normal subjects. Clin Auton Res 1995; 5(1):19-23.

[50] Milnor WR. Cardiovascular physiology. Oxford University Press, 1990.

[51] Huhta J, Moise K, Fisher D, Sharif D, Wasserstrum N, Martin C. Detection and quantitation of constriction of the fetal ductus arteriosus by doppler echocardiography. Circulation 1987; 75(2):406-412.

[52] de Marchi SF, Bodenmüller M, Lai DL, Seiler C. Pulmonary venous flow velocity patterns in 404 individuals without cardiovascular disease. Heart 2001; 85(1):23-29.

[53] Mauroy B, Filoche M, Andrade JS, Sapoval B. Interplay between geometry and flow distribution in an airway tree. Phys Rev Lett 2003; 90(14):148 101.

[54] Smolich JJ, Kenna KR, Cheung MM. Onset of asphyxial state in non-respiring interval between cord clamping and ventilation increases hemodynamic lability of birth transition in preterm lambs. J Appl Physiol 2015; .

[55] Kilner PJ, Balossino R, Dubini G, Babu-Narayan SV, Taylor AM, Pennati G, Migliavacca F. Pulmonary regurgitation: The effects of varying pulmonary artery compliance, and of increased resistance proximal or distal to the compliance. Int J Cardiol 2009; 133(2):157-166.

[56] Kiser CC, Handy TA, Lemley EC, Papavassiliou DV, Neeman HJ. Reynolds number dependence for laminar flow loss coefficients in tee and wye junctions. ASME 2010 3rd Joint US-European Fluids Engineering Summer Meeting collocated with 8th International Conference on Nanochannels, Microchannels, and Minichannels, American Society of Mechanical Engineers, 2010; 341-347.

[57] Jamison DK, Villemonte JR. Junction losses in laminar and transitional flows. Journal of the Hydraulics Division 1971; 97(7):1045-1063.

[58] Serre M, Odgaard A, Elder R. Energy loss at combining pipe junction. J Hydraul Eng 1994; 120(7):808-830. 


\section{University Library}

\section{- M M N E R VA A gateway to Melbourne's research publications}

Minerva Access is the Institutional Repository of The University of Melbourne

Author/s:

Mynard, JP;Valen-Sendstad, K

Title:

A unified method for estimating pressure losses at vascular junctions

Date:

2015-07-01

Citation:

Mynard, J. P. \& Valen-Sendstad, K. (2015). A unified method for estimating pressure losses at vascular junctions. INTERNATIONAL JOURNAL FOR NUMERICAL METHODS IN BIOMEDICAL ENGINEERING, 31 (7), https://doi.org/10.1002/cnm.2717.

Persistent Link:

http://hdl.handle.net/11343/124212 